\title{
Detecting Covariance Symmetries in Polarimetric SAR Images
}

\author{
Luca Pallotta, Member, IEEE, Carmine Clemente, Member, IEEE, \\ Antonio De Maio, Fellow, IEEE, and John J. Soraghan, Senior Member, IEEE
}

\begin{abstract}
The availability of multiple images of the same scene acquired with the same radar but with different polarizations, both in transmission and reception, has the potential to enhance the classification, detection, and/or recognition capabilities of a remote sensing system. A way to take advantage of the fullpolarimetric data is to extract, for each pixel of the considered scene, the polarimetric covariance matrix, the coherence matrix, and the Muller matrix and to exploit them in order to achieve a specific objective. A framework for detecting covariance symmetries within polarimetric synthetic aperture radar (SAR) images is here proposed. The considered algorithm is based on the exploitation of special structures assumed by the polarimetric coherence matrix under symmetrical properties of the returns associated with the pixels under test. The performance analysis of the technique is evaluated on both simulated and real L-band SAR data, showing a good classification level of the different areas within the image.
\end{abstract}

Index Terms-Coherence and covariance scattering matrix, polarimetric SAR image, radar image classification, synthetic aperture radar (SAR).

\section{INTRODUCTION}

$\mathbf{P}$ OLARIMETRIC synthetic aperture radar (SAR) imaging and the information obtainable from this kind of sensor configuration have attracted great interest from the research and end-user communities in recent years. Benefits, provided by the availability of multiple images of the same scene acquired with the same radar but with different polarizations, both in transmission and reception $(\mathrm{HH}, \mathrm{HV}$, and VV), include enhancing the classification, detection, and/or recognition ca-

Manuscript received October 29, 2015; revised February 22, 2016, March 29, 2016, April 22, 2016, and June 27, 2016; accepted July 22, 2016. Date of publication October 19, 2016; date of current version November 29, 2016. This work was supported in part by the Italian Ministry of Education, University and Research (MIUR) within the framework of project PON03PE_00159_6 "MoDiSTA — Soluzioni innovative per il Monitoraggio e la Diagnostica preventiva di infrastrutture e flotte di veicoli da remoto al fine di elevare i livelli di disponibilità, efficienza e sicurezza dei siSTemi ferroviAri," by the Engineering and Physical Sciences Research Council under Grant EP/K014307/1, and by the MOD University Defence Research Collaboration in Signal Processing.

L. Pallotta was with the Centro Regionale Information Communication Technology (CeRICT) scrl, Cinthia c/o Complesso Universitario di Monte Sant'Angelo, 80126 Napoli, Italy. He is now with the Università degli Studi di Napoli "Federico II," 80125 Napoli, Italy (e-mail: luca.pallotta@ unina.it).

C. Clemente and J. J. Soraghan are with the Centre for Excellence in Signal and Image Processing, University of Strathclyde, Glasgow G1 1XW, U.K. (e-mail: carmine.clemente@strath.ac.uk; j.soraghan@strath.ac.uk).

A. De Maio is with the Dipartimento di Ingegneria Elettrica e delle Tecnologie dell'Informazione, Università degli Studi di Napoli "Federico II," 80125 Napoli, Italy (e-mail: ademaio@unina.it).

Color versions of one or more of the figures in this paper are available online at http://ieeexplore.ieee.org.

Digital Object Identifier 10.1109/TGRS.2016.2595626 pabilities of the entire system. Among the different techniques available in open literature [1], a possible approach is to take advantage of the full-polarimetric data extracting for each pixel of the considered scene the polarimetric covariance matrix, coherence matrix, Muller matrix, and so on [1]-[4], and to use them in order to achieve a specific objective. Usually, the quantity measured by a polarimetric radar is the wellknown scattering matrix [3] (also called the Sinclair matrix $[1$, p. 63]); however, it is very useful to express the latter in a vectorized form and compute some second-order momentbased metrics, i.e., covariance and coherence matrices, that can be utilized to have inference about the scattering mechanisms characterizing the objects in the scene of interest. Moreover, a widely accepted processing strategy to deal with polarimetric SAR images relies on the coherent decomposition of the polarimetric scattering matrix. In this context, the Pauli [5], Krogager [6], and Cameron [7] decompositions play a central role. The aim of all these decompositions is to represent the scattering matrix as a combination of the scattering responses of independent elements (for instance, single/oddbounce scattering and double/even-bounce scattering), to associate a physical mechanism with each component and to extract relevant characteristics from polarimetric data sets.

An example of application of polarimetry can be found in [8], where the coherence matrix is exploited for extracting average parameters from experimental data. The algorithm is based on a second-order statistical model that does not require any specification of the underlying multivariate statistical distribution. In fact, it makes the assumption that, in each cell, there is always a dominant average scattering mechanism, and then, the parameters of this average component are estimated and related to the physical structures of the observed objects. Another example can be found in [9], where the use of both amplitude and phase information of the $\mathrm{HH}, \mathrm{HV}$, and $\mathrm{VV}$ images is introduced to distinguish among different scattering behaviors. By doing so, it is possible to interpret radar images and, in addition, to provide information aiding surface characterization through modeling of the polarimetric response of different types of terrain. In [10], Park et al. proposed a new model for vegetation scattering mechanisms of mountainous forests, extending the classic radiative transfer model and taking into account the sloping ground surface under vegetation canopy. Finally, many other works in the last few years use polarimetry: for oil spill detection [11], [12], for ice thickness retrieval [13], and for feature detection within a SAR image [14].

In this paper, we propose and analyze a framework for detecting covariance symmetries within a polarimetric SAR 
image, through the exploitation of special structures assumed by the covariance (and consequently by the coherence) matrix under symmetrical properties of the returns associated to the pixels under analysis. Since our problem is formulated in terms of a composite hypothesis test including nested instances, the classic generalized maximum-likelihood (GML) approach [15] does not prove useful. In fact, it always leads to the selection of the hypothesis with the higher degree of uncertainty and which incorporates the nested instances [15], [16]. To circumvent this drawback, it is paramount to consider a modified version of the GML to accommodate nested signal models. Specifically, it is necessary to add to the GML (under each hypothesis) a penalty term related to the number of parameters to estimate; this leads to the so-called model order selection techniques [17]-[20] to classify each pixel on the base of the corresponding coherence matrix structure. The knowledge of the symmetry would then allow enhanced performance for applications such as the knowledge-based Ground Moving Target Indicator, oil spill detection, and land cover classification.

The remainder of this paper is organized as follows. In Section II, the problem is introduced, whereas in Section III, the proposed framework for detecting covariance symmetries is developed. The performance of the proposed technique applied on simulated and real L-band SAR data is presented and discussed in Section IV. Finally, in Section V, some concluding remarks are given.

Notation: We adopt the notation of using boldface for vectors $\boldsymbol{a}$ (lowercase) and matrices $\boldsymbol{A}$ (uppercase). The conjugate and conjugate transpose operators are denoted by the symbols $(\cdot)^{*}$ and $(\cdot)^{\dagger}$, respectively, whereas the symbol $(\cdot)^{+}$denotes the pseudoinverse. $\operatorname{tr}\{\cdot\}$ and $\operatorname{det}(\cdot)$ are, respectively, the trace and the determinant of the square matrix argument. $\boldsymbol{I}$ and $\mathbf{0}$ denote, respectively, the identity matrix and the matrix with zero entries (their size is determined from the context). diag $(\boldsymbol{a})$ indicates the diagonal matrix whose $i$ th diagonal element is the $i$ th entry of $\boldsymbol{a}$. The letter $j$ represents the imaginary unit (i.e., $j=\sqrt{-1}$ ). For any complex number $x,|x|$ represents the modulus of $x, \operatorname{Re}\{x\}$ is its real part, and $\operatorname{Im}\{x\}$ is its imaginary part. Moreover, $\mathcal{H}_{n}^{++}$is the set of $n \times n$ positive semidefinite (psd) Hermitian matrices, $\mathcal{S}_{n}^{++}$is the set of $n \times n$ psd symmetric matrices, and $\mathcal{P}_{n}^{++}$is the set of $n \times n$ real psd persymmetric matrices. ${ }^{1}$ Finally, $\mathbb{E}[\cdot]$ denotes statistical expectation.

\section{Parameter Definition And Datacube CONSTRUCTION}

A multipolarization SAR sensor, for each pixel of the image under test, measures $N=3$ complex returns, which are collected from three different polarimetric channels (namely,

\footnotetext{
${ }^{1}$ A real persymmetric matrix $\boldsymbol{C}_{n}$ is a matrix with the following property $\boldsymbol{C}_{n}=\boldsymbol{J} \boldsymbol{C}_{n}^{T} \boldsymbol{J}$, with $\boldsymbol{J}$ as the $n \times n$ permutation matrix
}

$$
\boldsymbol{J}=\left[\begin{array}{cccccc}
0 & 0 & \cdots & 0 & 0 & 1 \\
0 & 0 & \cdots & 0 & 1 & 0 \\
0 & 0 & \cdots & 1 & 0 & 0 \\
\vdots & \vdots & & \vdots & \vdots & \vdots \\
0 & 1 & \cdots & 0 & 0 & 0 \\
1 & 0 & \cdots & 0 & 0 & 0
\end{array}\right] .
$$

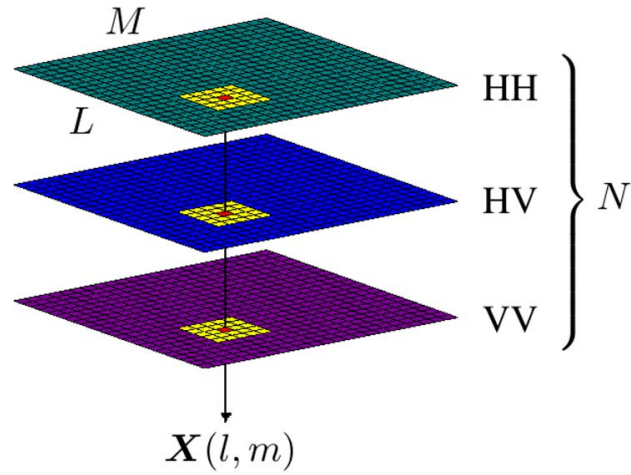

Fig. 1. Pictorial representation of constructing the datacube for polarimetric images.

$\mathrm{HH}, \mathrm{HV}$, and VV). The $N$ returns associated with the same pixel are organized in the specific order $\mathrm{HH}, \mathrm{HV}$, and VV to form the vector $\boldsymbol{X}(l, m), l=1, \ldots, L$ and $m=1, \ldots, M$ ( $L$ and $M$ represent the vertical and horizontal sizes of the image, respectively). Therefore, the sensor provides a 3-D data stack $\boldsymbol{X}$ of size $M \times L \times N$, which is referred to as the datacube in the following, whose pictorial representation is given in Fig. 1.

Starting from the datacube $\boldsymbol{X}$ of the scene illuminated by the radar, for each pixel under test, we extract a rectangular neighborhood $\mathcal{A}$ of size $K=W_{1} \times W_{2} \geq N$. We denote by $\boldsymbol{R}$ the matrix whose columns are the vectors of the polarimetric returns from the pixels of $\boldsymbol{X}$ that fall in region $\mathcal{A}$. Matrix $\boldsymbol{R}$ is modeled as a random matrix, whose columns are assumed to be statistically independent and identically distributed random vectors drawn from a complex circular zero-mean Gaussian distribution with positive definite covariance matrix $C$.

\section{Covariance Symmetry Detection}

The framework proposed in this paper uses the special structures assumed by the covariance and, consequently, by the corresponding coherence matrix over objects that scatter with a specific symmetry property [1, pp. 69-72], [2]. In this section, we illustrate such structures arising when some important scattering symmetric properties become predominant, and then, we introduce some algorithms that allow the detection of the specific covariance symmetry.

We consider the polarimetric covariance matrix in the presence of a reciprocal medium [1], [2], which is a $3 \times 3$ Hermitian matrix, i.e.,

$$
\boldsymbol{C}=\left[\begin{array}{lll}
c_{\mathrm{hhhh}} & c_{\mathrm{hhhv}} & c_{\mathrm{hhvv}} \\
c_{\mathrm{hhhv}}^{*} & c_{\mathrm{hvhv}} & c_{\mathrm{hvvv}} \\
c_{\mathrm{hhvv}}^{*} & c_{\mathrm{hvvv}}^{*} & c_{\mathrm{vvvv}}
\end{array}\right]
$$

completely described by $n=9$ real scalar values. In fact, the diagonal entries of this matrix are the conventional backscattering coefficients, which are real quantities, whereas the off-diagonal elements are complex values.

Let us consider now the presence of a reflection symmetry with respect to a vertical plane. It is usually observed over horizontal natural environments and produces complete decorrelations between the copolarized and the cross-polarized elements (the details regarding the proof of this covariance 
structure can be found in [2]). Consequently, the covariance matrix under reflection symmetry assumes the following special form, which is fully described by $n=5$ real scalar values, i.e.,

$$
\boldsymbol{C}=\left[\begin{array}{ccc}
c_{\mathrm{hhhh}} & 0 & c_{\mathrm{hhvv}} \\
0 & c_{\mathrm{hvhv}} & 0 \\
c_{\mathrm{hhvv}}^{*} & 0 & c_{\mathrm{vvvv}}
\end{array}\right]
$$

From a physical point of view, this result is valid for volume scattering, surface scattering, or volume-surface interactions to all scattering orders or to the total scattering effects no matter how dense is the medium or how rough is the surface as long as the scattering configuration has the reflection symmetry [2].

Let us consider now the presence of a rotation symmetry, which is characterized by a covariance matrix invariant under the rotation around an axis by any considered angle [2]. Consequently, the polarimetric covariance matrix assumes the following special structure, completely described by $n=3$ real scalar values, i.e.,

$$
\boldsymbol{C}=\left[\begin{array}{ccc}
c_{\mathrm{hhhh}} & c_{\mathrm{hhhv}} & c_{\mathrm{hhvv}} \\
-c_{\mathrm{hhhv}} & c_{\mathrm{hvhv}} & c_{\mathrm{hhhv}} \\
c_{\mathrm{hhvv}} & -c_{\mathrm{hhhv}} & c_{\mathrm{hhhh}}
\end{array}\right]
$$

where $c_{\mathrm{hhhv}}$ is purely imaginary, $c_{\mathrm{hhvv}}$ is purely real, and $c_{\text {hvhv }}=\left(c_{\text {hhhh }}-c_{\text {hhvv }}\right) / 2$. For instance, a chiral medium made by embedding helixes in an isotropic background can be considered as having both reciprocity and rotation symmetry [2].

Finally, the azimuth symmetry arises as the combination of a rotation symmetry with a reflection symmetry in any plane that contains the rotation symmetry axis. It can be observed for dense volumetric environments [3], and the corresponding polarimetric covariance matrix shares the following form:

$$
\boldsymbol{C}=\left[\begin{array}{ccc}
c_{\mathrm{hhhh}} & 0 & c_{\mathrm{hhvv}} \\
0 & c_{\mathrm{hvhv}} & 0 \\
c_{\mathrm{hhvv}} & 0 & c_{\mathrm{hhhh}}
\end{array}\right]
$$

where $c_{\mathrm{hvhv}}=\left(c_{\mathrm{hhhh}}-c_{\mathrm{hhvv}}\right) / 2$. Evidently, (4) is entirely described by $n=2$ real scalar values.

With the above models for the polarimetric covariance matrix structure, we focus on the problem of data classification exploiting the scattering properties of the pixel under test and of a set of neighborhood pixels. Specifically, we associate to each pixel an "average" or "dominant" symmetry property on the base of the specific structure assumed by its covariance matrix. Moreover, to simplify the analytic tractability, we move to a transformed matrix domain (as described in Lemma 3.1) where some redundant information (contained in the functionally dependent coefficients of the covariance) translates into zeros of the corresponding transformed matrix. Before proceeding further, it is necessary to define the multiple hypotheses associated to the problem under consideration, i.e.,

$$
\begin{cases}H_{1}: & \text { no symmetry } \\ H_{2}: & \text { reflection symmetry } \\ H_{3}: & \text { rotation symmetry } \\ H_{4}: & \text { azimuth symmetry. }\end{cases}
$$
$\boldsymbol{S}_{0}=\boldsymbol{R} \boldsymbol{R}^{\dagger}$ is a sufficient statistic for $\boldsymbol{C}$.

Hence, in Lemma 3.1, we show how to compute the transformed matrix from the covariance and determine its structure under the hypotheses $H_{1}, \ldots, H_{4}$.

Lemma 3.1: Let us denote by

$$
\begin{aligned}
& \boldsymbol{U}=\left[\begin{array}{lll}
1 & 0 & 0 \\
0 & 0 & 1 \\
0 & 1 & 0
\end{array}\right] \\
& \boldsymbol{E}=\left[\begin{array}{ccc}
1 & 0 & 0 \\
0 & \frac{1}{\sqrt{2}} & 0 \\
0 & 0 & 1
\end{array}\right] \\
& \boldsymbol{T}=\frac{1}{\sqrt{2}}\left[\begin{array}{ccc}
1 & 0 & 1 \\
1 & 0 & -1 \\
0 & \sqrt{2} & 0
\end{array}\right] \\
& \boldsymbol{V}=\left[\begin{array}{ccc}
1 & 0 & 0 \\
0 & 0 & j \\
0 & 1 & 0
\end{array}\right]
\end{aligned}
$$

four transformation matrices, where $\boldsymbol{U}$ is orthogonal, whereas $\boldsymbol{V}$ and $\boldsymbol{T}$ are unitary.

Then, under the reflection symmetry hypothesis, we have

$$
\boldsymbol{U} \boldsymbol{C} \boldsymbol{U}^{\dagger}=\left[\begin{array}{ccc}
c_{\mathrm{hhhh}} & c_{\mathrm{hhvv}} & 0 \\
c_{\mathrm{hhvv}}^{*} & c_{\mathrm{vvvv}} & 0 \\
0 & 0 & c_{\mathrm{hvhv}}
\end{array}\right]=\left[\begin{array}{cc}
\boldsymbol{C}_{1} & \mathbf{0} \\
\mathbf{0} & c
\end{array}\right]
$$

where $C_{1} \in \mathcal{H}_{2}^{++}$, and $c$ is a positive real number.

Under the rotation symmetry hypothesis, we have

$$
\begin{aligned}
\boldsymbol{V} \boldsymbol{E} \boldsymbol{T} \boldsymbol{C} \boldsymbol{T}^{\dagger} \boldsymbol{E} \boldsymbol{V}^{\dagger} & =\left[\begin{array}{ccc}
c_{\mathrm{hhhh}}+c_{\mathrm{hhvv}} & 0 & 0 \\
0 & c_{\mathrm{h} h \mathrm{hv}} & \operatorname{Im}\left\{c_{\mathrm{hhhv}}\right\} \\
0 & \operatorname{Im}\left\{c_{\mathrm{hhhv}}\right\} & c_{\mathrm{hvhv}}
\end{array}\right] \\
& =\left[\begin{array}{cc}
a & \mathbf{0} \\
\mathbf{0} & \boldsymbol{C}_{2}
\end{array}\right]
\end{aligned}
$$

where $C_{2} \in \mathcal{P}_{2}^{++}$, and $a$ is a positive real number.

Finally, under the azimuth symmetry hypothesis, we have

$$
\begin{aligned}
& \boldsymbol{E} \boldsymbol{T} \boldsymbol{C} \boldsymbol{T}^{\dagger} \boldsymbol{E}=\left[\begin{array}{ccc}
c_{\mathrm{hhhh}}+c_{\mathrm{hhvv}} & 0 & 0 \\
0 & c_{\mathrm{hvhv}} & 0 \\
0 & 0 & c_{\mathrm{hvhv}}
\end{array}\right] \\
& =\left[\begin{array}{lll}
a & 0 & 0 \\
0 & b & 0 \\
0 & 0 & b
\end{array}\right]
\end{aligned}
$$

where $b$ is a positive real number.

Proof: The proof is omitted since it can be obtained by performing the matrix multiplications on the right-hand side of (6)-(8).

Let us consider the complex multivariate probability density function (pdf) of the observable matrix $\boldsymbol{R}$, i.e.,

$$
f_{\boldsymbol{R}}(\boldsymbol{R} \mid \boldsymbol{C})=\frac{1}{\pi^{3 K}[\operatorname{det}(\boldsymbol{C})]^{K}} \exp \left\{-\operatorname{tr}\left(\boldsymbol{C}^{-1} \boldsymbol{R} \boldsymbol{R}^{\dagger}\right)\right\}
$$

the Fisher-Neyman factorization theorem [15] implies that 
Now, the maximum-likelihood (ML) estimate of $\boldsymbol{C}$ can be obtained as the optimal solution to the optimization problem, i.e.,

$$
\begin{array}{r}
\max _{\boldsymbol{C}} \log \left(f_{\boldsymbol{R}}(\boldsymbol{R} \mid \boldsymbol{C})\right)=-K \min _{\boldsymbol{C}}\left[\log \operatorname{det}(\boldsymbol{C})+\operatorname{tr}\left(\boldsymbol{C}^{-1} \boldsymbol{S}\right)\right] \\
-3 K \log \pi
\end{array}
$$

where $\boldsymbol{S}=(1 / K) \boldsymbol{S}_{0}$.

The following proposition shows the form assumed by the optimal value of problem (10), as well as the ML optimizer, in the presence of the four different scattering symmetries previously described.

Proposition 3.2: The optimal value of

$$
\min _{\boldsymbol{C} \in \mathcal{H}_{3}^{++}}\left[\log \operatorname{det}(\boldsymbol{C})+\operatorname{tr}\left(\boldsymbol{C}^{-1} \boldsymbol{S}\right)\right]
$$

and the corresponding optimal solution $\breve{C}$ are respectively given by the following.

1) No symmetry:

$$
\begin{aligned}
& \log \operatorname{det}(\boldsymbol{S})+3 \\
& \breve{C}=\boldsymbol{S} .
\end{aligned}
$$

2) Reflection symmetry:

$$
\begin{aligned}
& \log \operatorname{det}\left(\overline{\boldsymbol{S}}_{1,1}\right)+\log \left(\bar{S}_{3,3}\right)+3 \\
& \breve{\boldsymbol{C}}=\boldsymbol{U}^{\dagger}\left[\begin{array}{cc}
\overline{\boldsymbol{S}}_{1,1} & \mathbf{0} \\
\mathbf{0} & \bar{S}_{3,3}
\end{array}\right] \boldsymbol{U}
\end{aligned}
$$

where $\overline{\boldsymbol{S}}=\boldsymbol{U} \boldsymbol{S} \boldsymbol{U}^{\dagger}=\left[\begin{array}{ll}\overline{\boldsymbol{S}}_{1,1} & \overline{\boldsymbol{S}}_{1,3} \\ \overline{\boldsymbol{S}}_{3,1} & \bar{S}_{3,3}\end{array}\right]$

3) Rotation symmetry:

$$
\begin{aligned}
& \log \operatorname{det}\left(\frac{1}{2}\left(\tilde{\boldsymbol{S}}_{2,2}+\boldsymbol{J} \tilde{\boldsymbol{S}}_{2,2} \boldsymbol{J}\right)\right)+\log \left(\tilde{S}_{1,1}\right)+3+\log 2 \\
& \breve{\boldsymbol{C}}=\boldsymbol{T}^{\dagger} \boldsymbol{E}^{-1} \boldsymbol{V}^{\dagger}\left[\begin{array}{cc}
\tilde{S}_{1,1} & \mathbf{0} \\
\mathbf{0} & \frac{1}{2}\left(\tilde{\boldsymbol{S}}_{2,2}+\boldsymbol{J} \tilde{\boldsymbol{S}}_{2,2} \boldsymbol{J}\right)
\end{array}\right] \boldsymbol{V} \boldsymbol{E}^{-1} \boldsymbol{T}
\end{aligned}
$$$$
\text { where } \tilde{\boldsymbol{S}}=\boldsymbol{V} \boldsymbol{E} \boldsymbol{T} \boldsymbol{S} \boldsymbol{T}^{\dagger} \boldsymbol{E} \boldsymbol{V}^{\dagger}=\left[\begin{array}{ll}
\tilde{S}_{1,1} & \tilde{\boldsymbol{S}}_{1,2} \\
\tilde{\boldsymbol{S}}_{2,1} & \tilde{\boldsymbol{S}}_{2,2}
\end{array}\right] .
$$

4) Azimuth symmetry:

$$
\begin{aligned}
& \log \left(\hat{S}_{1,1}\right)+2 \log \left(\frac{\hat{S}_{2,2}+\hat{S}_{3,3}}{2}\right)+3+\log 2 \\
& \breve{\boldsymbol{C}}=\boldsymbol{T}^{\dagger} \boldsymbol{E}^{-1} \operatorname{diag}\left(\hat{S}_{1,1}, \frac{\hat{S}_{2,2}+\hat{S}_{3,3}}{2}, \frac{\hat{S}_{2,2}+\hat{S}_{3,3}}{2}\right) \boldsymbol{E}^{-1} \boldsymbol{T}
\end{aligned}
$$

where $\hat{\boldsymbol{S}}=\boldsymbol{E} \boldsymbol{T} \boldsymbol{S} \boldsymbol{T}^{\dagger} \boldsymbol{E}$, and $\hat{S}_{1,1}, \hat{S}_{2,2}$, and $\hat{S}_{3,3}$ are its diagonal entries.

Proof: See Appendix A.

\section{A. Model Order Selection}

As previously discussed, we are focused on a composite multiple-hypothesis testing problem that includes both nested and non-nested instances. The GML approach does not appear useful for this study since the likelihood always assumes the highest value under the $H_{1}$ hypothesis. Hence, to overcome this problem, we need to consider modified versions of the GML to accommodate nested instances. Specifically, a penalty function that is dependent on the number of unknown elements to estimate is added to the GML under each hypothesis. This leads to the so-called model order selectors ${ }^{2}[17]-[20]$ that allow us to estimate the correct structure (the model order) from the available observables ( $\boldsymbol{R}$ in this case). Following the above guidelines, it is necessary to evaluate a decision statistic under each hypothesis, and then, the order is chosen as the one which corresponds to the minimum among the four statistics. Before proceeding further, it is worth underlying that model order selection approaches have been already used in SAR remote sensing applications. In this context, we mention the works in [21]-[24] for interferometric SAR and that in [25] with reference to double-scatterer detection in SAR tomography.

The general order selection rules proposed in [17] and [18] can be expressed through the corresponding decision statistics in the following compact form:

$$
-2 \log \left(f\left(\boldsymbol{R} \mid \breve{C}^{(n)}\right)\right)+n \eta(n, K)
$$

with $\breve{\boldsymbol{C}}^{(n)}$ the ML estimate of $\boldsymbol{C}$ comprising of $n$ parameters. The term $n \eta(n, K)$ is called the penalty coefficient, and its role can be intuitively explained observing that the first term in (12) decreases with increasing $n$ (for nested models), whereas the second term increases. As a consequence, $n \eta(n, K)$ in (12) penalizes overfitting [18]. Different selection strategies diversify due to the definition of this quantity. Specifically, for each criterion, we have the following:

- Akaike Information Criterion (AIC): $\eta(n, K)=2$;

- Generalized Information Criterion (GIC): $\eta(n, K)=\rho+$ 1 , with $\rho$ as an integer number greater than or equal to 2 ;

- Bayesian Information Criterion (BIC): $\eta(n, K)=$ $\log (K)$.

For the case at hand, substituting (10), obtained as described in Proposition 3.2, in (12), it is not difficult to obtain the following decision statistic under each hypothesis (notice that, for simplicity, the dependence of $\eta$ from $n$ and $K$ is omitted in the following equations):

- $H_{1}$ :

$$
2 K \log \operatorname{det}(\boldsymbol{S})+6 K+6 K \log (\pi)+9 \eta
$$

- $\mathrm{H}_{2}$ :

$$
2 K \log \operatorname{det}\left(\overline{\boldsymbol{S}}_{1,1}\right)+2 K \log \left(\bar{S}_{3,3}\right)+6 K+6 K \log (\pi)+5 \eta
$$

\footnotetext{
${ }^{2}$ We recommend to the interested reader reference [18] for a methodological framework toward the definition of some model order selectors (together with the corresponding penalty terms) based on the Kullback-Leibler information criterion and the Bayesian theory.
} 
- $H_{3}$ :

$$
\begin{aligned}
2 K \log \operatorname{det}\left(\frac{1}{2}\left(\tilde{\boldsymbol{S}}_{2,2}+\boldsymbol{J} \tilde{\boldsymbol{S}}_{2,2} \boldsymbol{J}\right)\right)+2 K \log \left(\tilde{S}_{1,1}\right) \\
+6 K+2 K \log 2+6 K \log (\pi)+3 \eta
\end{aligned}
$$

- $H_{4}$ :

$$
\begin{aligned}
2 K \log \left(\hat{S}_{1,1}\right)+4 K \log \left(\frac{\hat{S}_{2,2}+\hat{S}_{3,3}}{2}\right)+6 K \\
+2 K \log (2)+6 K \log (\pi)+2 \eta .
\end{aligned}
$$

A remark is now necessary: For the BIC, there are two particular assumptions on the Fisher information matrix $\boldsymbol{F}$ of the estimation problem. They are referred to as regularity conditions, and their validity is shown in Appendix B.

The last decision criterion considered herein is referred to as the exponentially embedded families (EEF) approach; its general theoretical formulation is laid down in [20], i.e.,

$\operatorname{EEF}(i)=\left\{l_{G_{i}}(\boldsymbol{R})-n(i)\left[\log \left(\frac{l_{G_{i}}(\boldsymbol{R})}{n(i)}\right)+1\right]\right\} u\left(\frac{l_{G_{i}}(\boldsymbol{R})}{n(i)}-1\right)$

with

$$
l_{G_{i}}(\boldsymbol{R})=2 \log \left(\frac{f\left(\boldsymbol{R} ; \breve{\boldsymbol{C}}^{(n(i))}\right)}{f\left(\boldsymbol{R} ; \boldsymbol{C}^{(0)}\right)}\right), \quad i=1, \ldots, 4
$$

$n(i)$ as the number of unknown parameters under the $i$ th hypothesis, and $u(\cdot)$ as the Heaviside step function, i.e., $u(\cdot)=1$ if its argument is greater than 0 ; otherwise, it is 0 . To proceed further, it is necessary to evaluate the functions $l_{G_{i}}(\cdot), i=$ $1, \ldots, 4$, in correspondence of the four hypotheses previously defined, but before doing this, we need to introduce a dummy hypothesis, i.e., $H_{0}$, where there is no dependence on the unknown parameters. Specifically, let us define the covariance matrix under the $H_{0}$ hypothesis, $\boldsymbol{C}^{(0)}$ as the $N$-dimensional identity matrix, i.e., $\boldsymbol{C}^{(0)}=\boldsymbol{I}_{N}$. With this choice, the functions $l_{G_{i}}(\cdot), i=1, \ldots, 4$, become

- $H_{1}$ :

$$
l_{G_{1}}(\boldsymbol{R})=-2 K \log \operatorname{det}(\boldsymbol{S})-6 K+2 \operatorname{tr}\left(\boldsymbol{S}_{0}\right)
$$

- $\mathrm{H}_{2}$ :

$$
\begin{aligned}
l_{G_{2}}(\boldsymbol{R})=-2 K \log \operatorname{det}\left(\overline{\boldsymbol{S}}_{1,1}\right) & \\
- & 2 K \log \left(\bar{S}_{3,3}\right)-6 K+2 \operatorname{tr}\left(\boldsymbol{S}_{0}\right)
\end{aligned}
$$

- $H_{3}$ :

$$
\begin{aligned}
l_{G_{3}}(\boldsymbol{R}) & =-2 K \log \operatorname{det}\left(\frac{1}{2}\left(\tilde{\boldsymbol{S}}_{2,2}+\boldsymbol{J} \tilde{\boldsymbol{S}}_{2,2} \boldsymbol{J}\right)\right) \\
- & 2 K \log \left(\tilde{S}_{1,1}\right)-6 K-2 K \log (2)+2 \operatorname{tr}\left(\boldsymbol{S}_{0}\right)
\end{aligned}
$$

- $H_{4}$ :

$$
\begin{array}{r}
l_{G_{4}}(\boldsymbol{R})=-2 K \log \left(\hat{S}_{1,1}\right)-4 K \log \left(\frac{\hat{S}_{2,2}+\hat{S}_{3,3}}{2}\right) \\
-6 K-2 K \log (2)+2 \operatorname{tr}\left(\boldsymbol{S}_{0}\right) .
\end{array}
$$

Finally, for the AIC, BIC, and GIC, we select the hypothesis that corresponds to the minimum decision statistic, whereas for the EEF, we select that which corresponds to the maximum, i.e.,

$$
\begin{aligned}
& \hat{h}_{\mathrm{AIC}}=\arg \min _{h} \operatorname{AIC}(h) \\
& \hat{h}_{\mathrm{BIC}}=\arg \min _{h} \operatorname{BIC}(h) \\
& \hat{h}_{\mathrm{GIC}}=\arg \min _{h} \operatorname{GIC}(h) \\
& \hat{h}_{\mathrm{EEF}}=\arg \max _{h} \operatorname{EEF}(h)
\end{aligned}
$$

where $h=1, \ldots, 4$ is the index identifying the specific hypothesis. In other words, for each pixel under test, the selected structure is that associated to $H=H_{\hat{h}}$. For the sake of completeness and to help the reader grasp all the details on the proposed procedure, in Algorithm 1, we have explicitly reported all the basic steps required to classify each pixel of the image. It refers to the order selector EEF. However, it is possible to consider other cases too, just substituting the EEF with the AIC, the $\mathrm{BIC}$, or the GIC and computing the minimum in place of the maximum, i.e., (15)-(17) instead of (18).

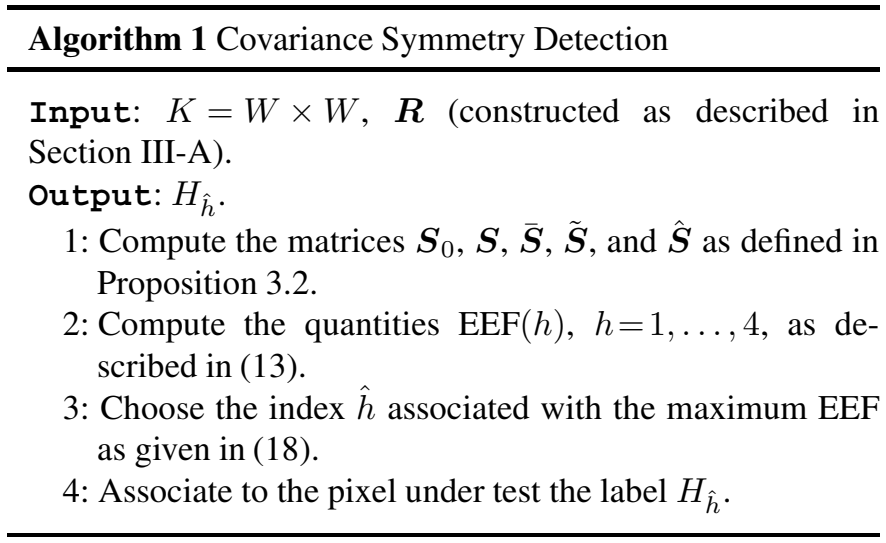

\section{RESULTS}

In this section, the performance of the proposed rules for covariance symmetry detection is assessed. In particular, to evaluate the effectiveness of the different techniques, both simulated and real radar data are considered.

\section{A. Analysis on Simulated Data}

In this section, the performance analysis on simulated data for the order selectors introduced in Section III-A is developed and discussed. In particular, the probability of correct classification is estimated (resorting to Monte Carlo simulations) 

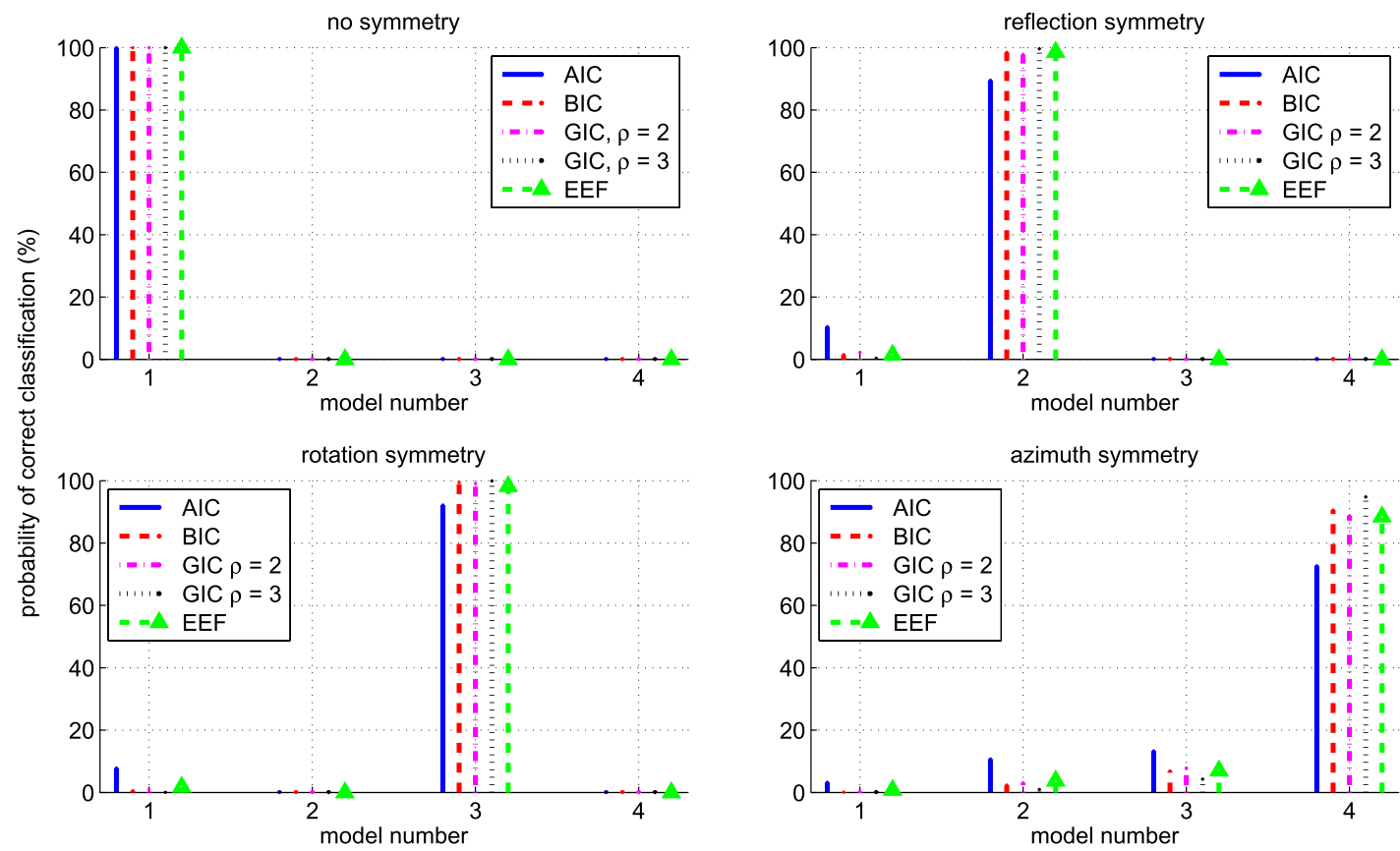

Fig. 2. Probability of correct classification (\%) for a simulated scenario with $K=25$ data and $M C=10^{4}$ Monte Carlo trials.

as the ratio between the number of correct classifications and the total number of trials $M C$, which is set to $10^{4}$. For each simulation run, $K$ 3-D zero-mean complex circular Gaussian vectors are simulated. Hence, they are colored to exhibit a covariance matrix structure characterizing a specific hypothesis of the testing problem. As to the theoretical covariance matrices defining the four scenarios (no symmetry, reflection, rotation, and azimuth symmetry), they are respectively given by

$$
\begin{aligned}
& \boldsymbol{C}_{1}=\left[\begin{array}{ccc}
1 & 0.2+0.3 j & 0.5-0.3 j \\
0.2-0.3 j & 0.25 & -0.2-0.2 j \\
0.5+0.3 j & -0.2+0.2 j & 0.8
\end{array}\right] \\
& \boldsymbol{C}_{2}=\left[\begin{array}{ccc}
1 & 0 & 0.5-0.3 j \\
0 & 0.25 & 0 \\
0.5+0.3 j & 0 & 0.4
\end{array}\right] \\
& \boldsymbol{C}_{3}=\left[\begin{array}{ccc}
1 & 0.3 j & 0.2 \\
-0.3 j & 0.4 & 0.3 j \\
0.2 & -0.3 j & 1
\end{array}\right] \\
& \boldsymbol{C}_{4}=\left[\begin{array}{ccc}
1 & 0 & 0.5 \\
0 & 0.25 & 0 \\
0.5 & 0 & 1
\end{array}\right] .
\end{aligned}
$$

In Fig. 2, the probability of correct classification (expressed in percentage) is reported for each of the four analyzed models, considering $K=25$ data vectors. The subplots refer, respectively, to the four considered covariance scenarios, and the performance measures are related to five order selectors, i.e., AIC, BIC, GIC with $\rho=2$, GIC with $\rho=3$, and EEF. The results show that the EEF, GIC, and BIC approaches provide a better performance than that achievable using the AIC. It is also worth observing that for small values of $K$, all the selectors exhibit a performance degradation due to the fact that the sample covariance matrix achieves good estimation performances only when $K$ is sufficiently higher than $N$. This estimation error becomes more impactful in cases where symmetries lead to similar structures in the covariance matrix. This situation arises with reference to the azimuth symmetry that shares a structure quite close to the rotation symmetry.

To study in detail the behavior of the proposed algorithm in the presence of a better and better covariance estimate, in Fig. 3, we report, for each selector, the probability of correct classification as a function of the number of looks, i.e., $K$. Again, the data are simulated as in Fig. 2, i.e., zero-mean complex circular Gaussian vectors with a covariance matrix given by $\boldsymbol{C}_{1}, \boldsymbol{C}_{2}, \boldsymbol{C}_{3}$, and $\boldsymbol{C}_{4}$, respectively. Moreover, $10^{4}$ Monte Carlo runs have been utilized.

From this analysis, it is clear that the case of symmetry absence is almost always correctly detected, independently of the utilized selector. Moreover, the azimuth symmetry case is the most challenging situation; in fact, only the GIC is able to ensure a probability of correct classification higher than $90 \%$ for small values of $K$. Finally, the curves show that the performances improve as $K$ increases, due to the better estimation of the covariance matrix owing to the greater number of available homogeneous data. This agrees with the intuition that the more available information on the scene, the better the algorithm performance.

To conclude the study on simulated data and to demonstrate a certain degree of robustness for the proposed algorithm with respect to the design hypotheses, we consider a mismodeling analysis where the data deviate from the Gaussian behavior; specifically, we model each column of matrix $\boldsymbol{R}$, e.g., $\boldsymbol{r}_{k}$ 

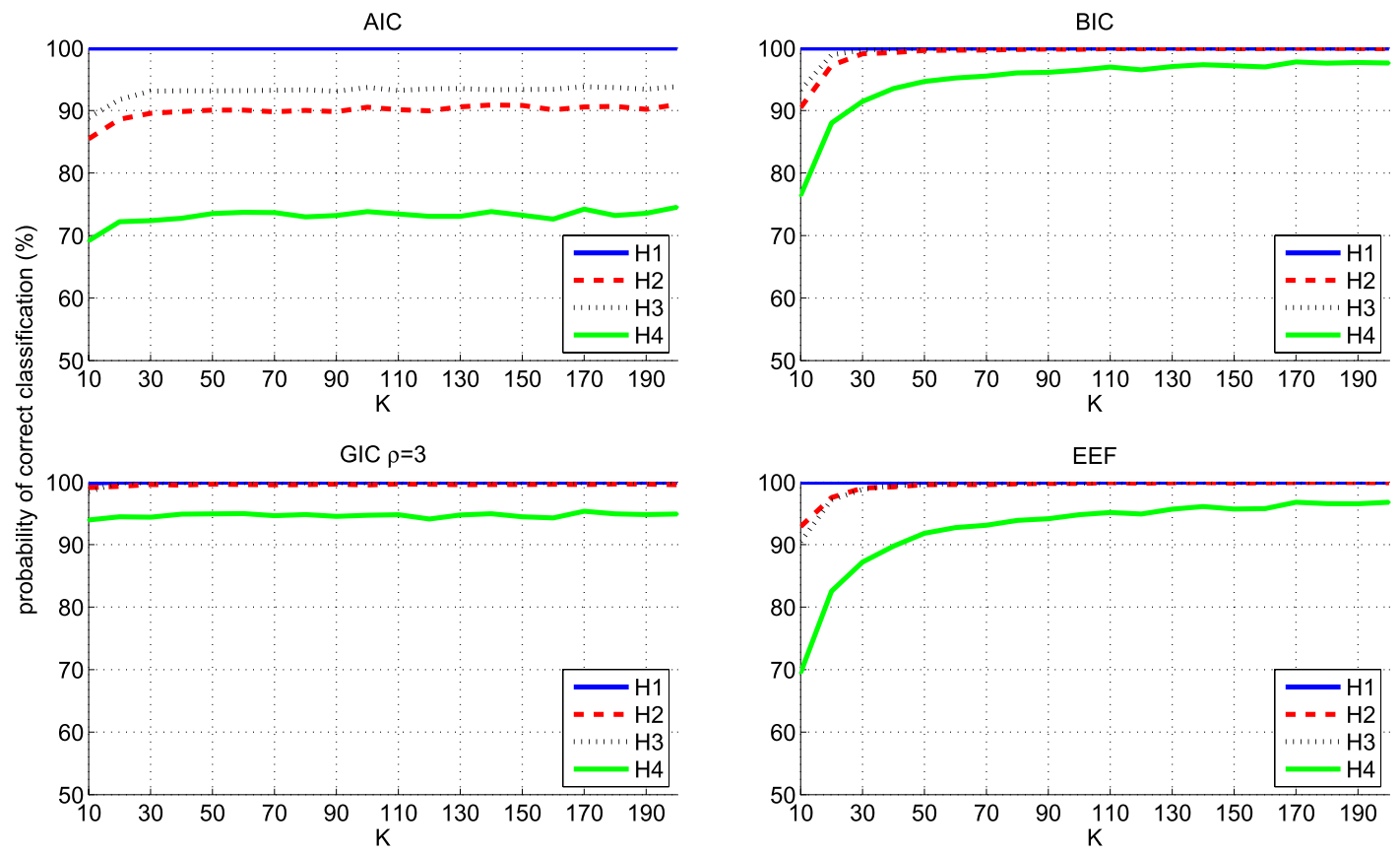

Fig. 3. Probability of correct classification (\%) versus the number of data $K$, with $M C=10^{4}$ Monte Carlo trials. The subplots refer to the different selectors, whereas the curves are related to the four considered covariance scenarios, i.e., $\boldsymbol{C}_{1}, \boldsymbol{C}_{2}, \boldsymbol{C}_{3}$, and $\boldsymbol{C}_{4}$.
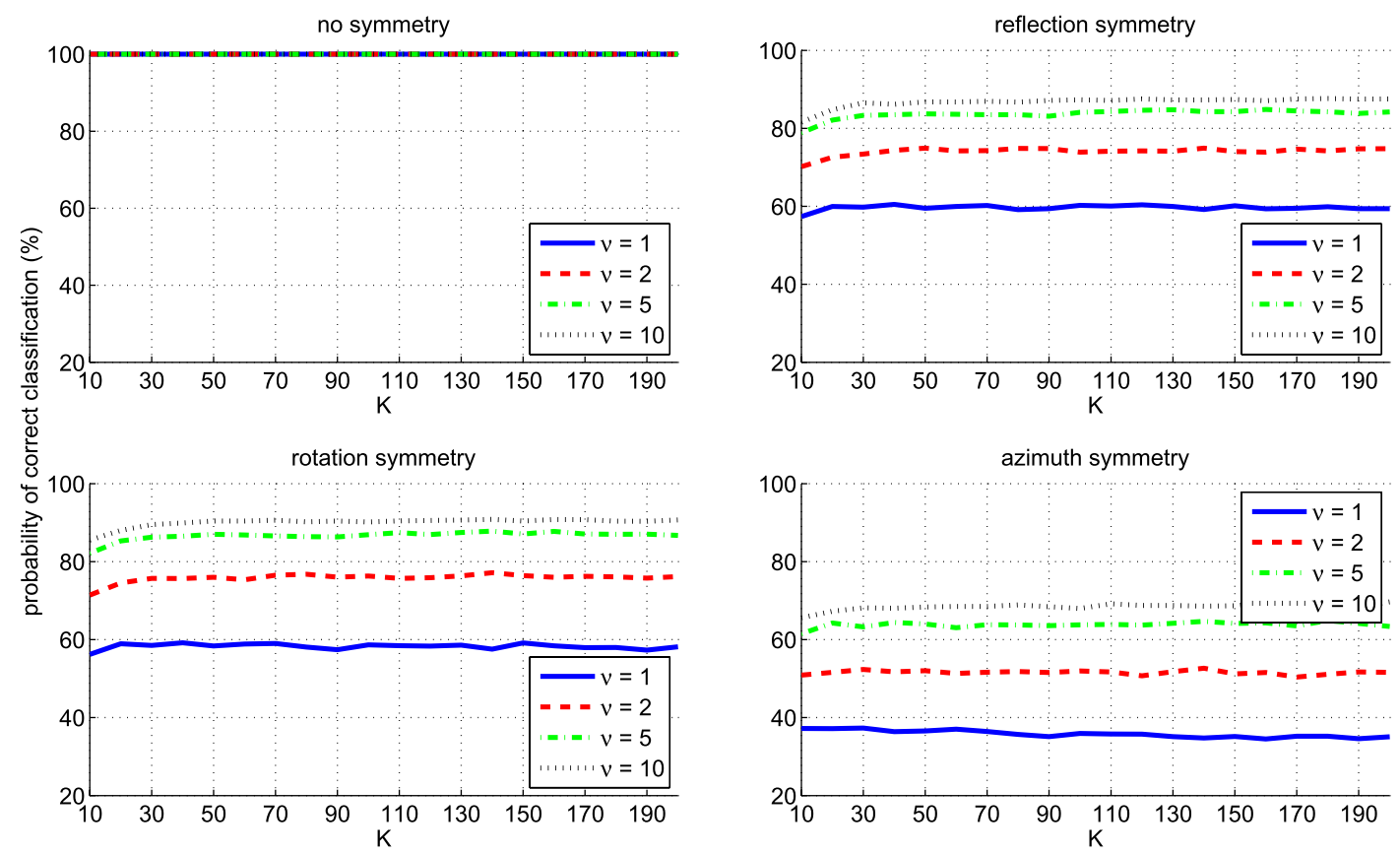

Fig. 4. Probability of correct classification (\%) for the AIC selector versus the number of data $K$ in a SIRV environment, with $M C=10^{4}$ Monte Carlo trials. The subplots refer to the different covariance scenarios, i.e., $\boldsymbol{C}_{1}, \boldsymbol{C}_{2}, \boldsymbol{C}_{3}$, and $\boldsymbol{C}_{4}$, whereas the curves refer to the four considered values of $\nu$.

$k=1, \ldots, K$, as a spherically invariant random vector (SIRV) [26]-[28], which can be written in the following form:

$$
\boldsymbol{r}_{k}=\sqrt{\tau_{k}} \boldsymbol{g}_{k}, \quad k=1, \ldots, K
$$

where $\tau_{k}$ is a positive real random variable (usually known as texture), and $\boldsymbol{g}_{k}$ is an $N$-dimensional zero-mean complex circular Gaussian vector, whose covariance matrix is set according to the four considered symmetry hypotheses, i.e., $C_{1}$,
$C_{2}, C_{3}$, and $C_{4}$, respectively. In the simulations, we assume that $\tau_{1}, \tau_{2}, \ldots, \tau_{K}$ are statistically independent. Moreover, they follow the Gamma distribution, i.e.,

$$
f(x)=\frac{1}{\Gamma(\nu)} \frac{1}{\mu^{\nu}} x^{\nu-1} e^{-x / \mu} u(x)
$$

where $\Gamma(\cdot)$ is the Eulerian Gamma function, and $\mu$ and $\nu>0$ are the scale and shape parameters, respectively (we set $\mu=1 / \nu$ to 

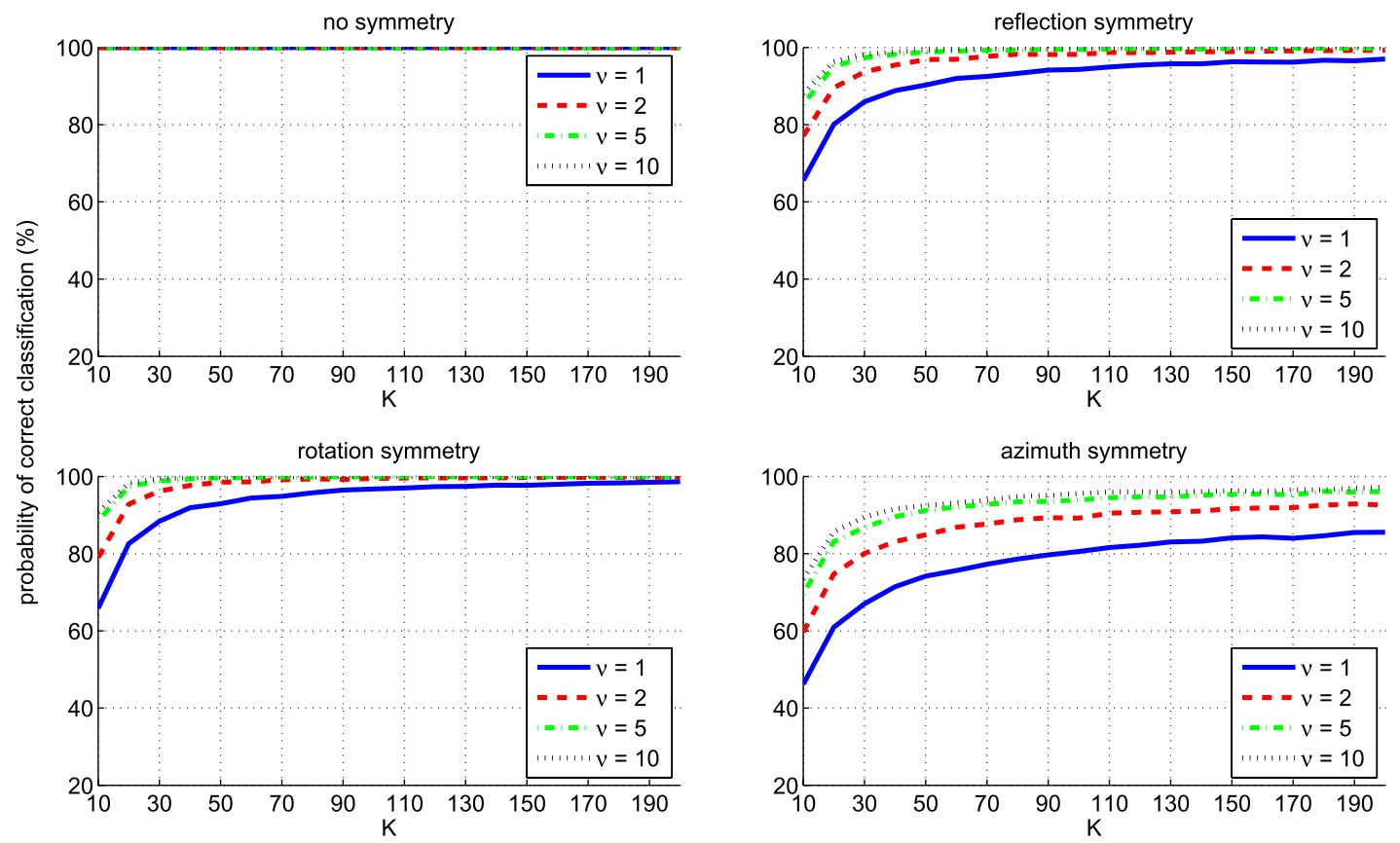

Fig. 5. Probability of correct classification (\%) for the BIC selector versus the number of data $K$ in a SIRV environment, with $M C=10^{4}$ Monte Carlo trials. The subplots refer to the different covariance scenarios, i.e., $\boldsymbol{C}_{1}, \boldsymbol{C}_{2}, \boldsymbol{C}_{3}$, and $\boldsymbol{C}_{4}$, whereas the curves refer to the four considered values of $\nu$.
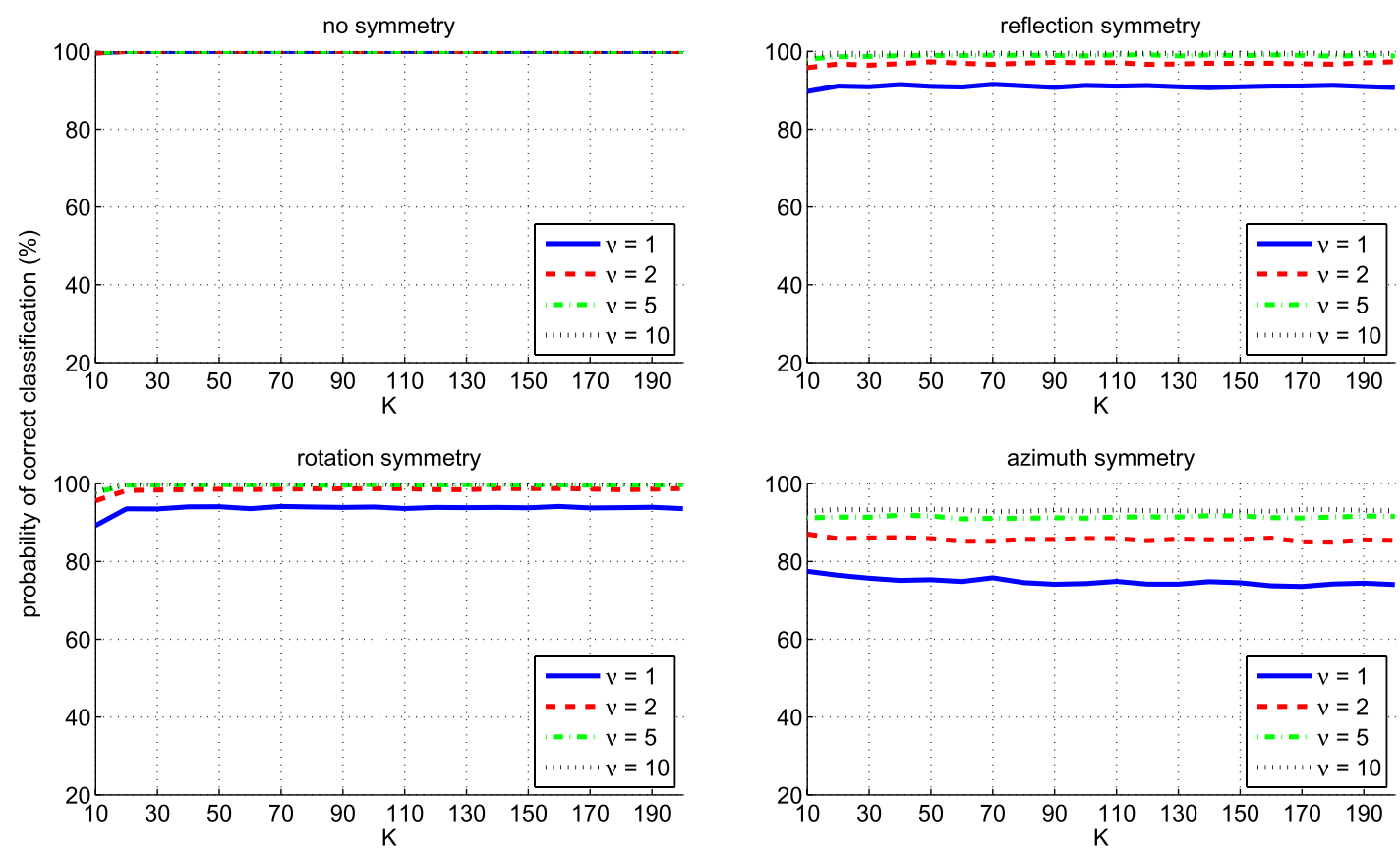

Fig. 6. Probability of correct classification (\%) for the GIC (with $\rho=3$ ) selector versus the number of data $K$ in a SIRV environment, with $M C=10^{4}$ Monte Carlo trials. The subplots refer to the different covariance scenarios, i.e., $\boldsymbol{C}_{1}, \boldsymbol{C}_{2}, \boldsymbol{C}_{3}$, and $\boldsymbol{C}_{4}$, whereas the curves refer to the four considered values of $\nu$.

have a Gamma distribution with unit mean). The adopted model for the textures implies that the amplitude pdfs of $\boldsymbol{r}_{1}, \ldots, \boldsymbol{r}_{K}$ are $K$-distributed. The analysis is conducted for different values of the shape parameter, i.e., $\nu=(1,2,5,10)$, and the results are reported in Figs. 4-7.

The curves clearly show that the GIC-, BIC-, and EEF-based detectors outperform the AIC. Moreover, the classification in the fourth hypothesis still remains a challenge as the structure of $\boldsymbol{C}_{4}$ is quite close to that of $\boldsymbol{C}_{3}$. Nevertheless, this mismodeling analysis has highlighted that even in the presence of data that do not comply with Gaussianity, the proposed algorithm shares some robustness and is able to grant satisfactory symmetry classification performances.

\section{B. Analysis on Real UAVSAR Data}

In this section, the results on real SAR data are shown and discussed. In particular, an L-band coherent polarimetric 

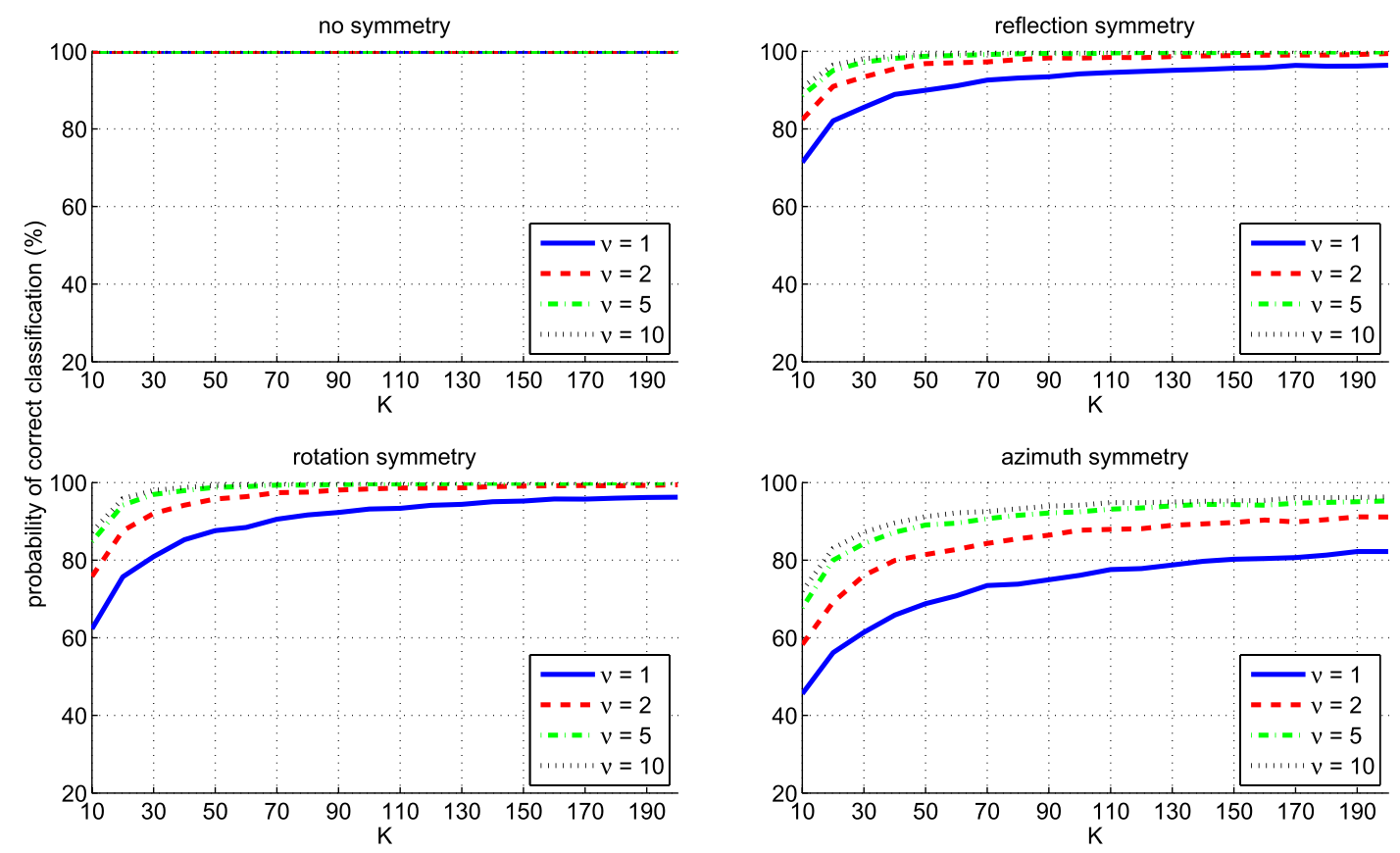

Fig. 7. Probability of correct classification (\%) for the EEF selector versus the number of data $K$ in a SIRV environment, with $M C=10^{4}$ Monte Carlo trials. The subplots refer to the different covariance scenarios, i.e., $\boldsymbol{C}_{1}, \boldsymbol{C}_{2}, \boldsymbol{C}_{3}$, and $\boldsymbol{C}_{4}$, whereas the curves refer to the four considered values of $\nu$.

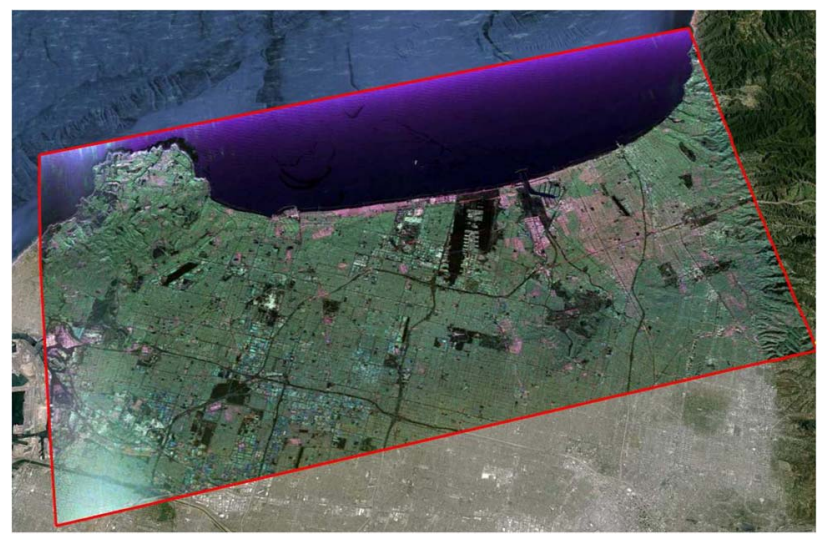

Fig. 8. Three-polarization color overlay of the UAVSAR pass SSurge_15305_14170_007_141120_L090_CX_01.

data set $^{3}$ acquired using uninhabited aerial vehicle SAR (UAVSAR) [29] on the Southern California Coast on November 20, $2014^{4}$ is utilized. The latter contains a scene acquired with three polarizations (HH, HV, and VV) and whose polarimetric overlay is shown in Fig. 8.

For our analysis, the selected area of interest is a subimage of $2000 \times 2000$ pixels (i.e., $L=M=2000$ ) containing both terrain and sea data. For comparison purposes, the span $[1$, p. 61] of such image (expressed in decibels) is reported in Fig. 9(a), whereas in Fig. 9(b), it is represented as its $H-A-\alpha$ decomposition [8] in RGB colors.

In Fig. 10, the detected symmetries for the reference image are plotted, using the AIC, BIC, GIC (with $\rho=3$ ), and EEF, respectively, with $K=25$, i.e., a $5 \times 5$ sliding window is utilized. Specifically, for each pixel of the considered scene, a specific color is indicated, which is associated to the specific hypothesis chosen by the test.

The results show that the AIC is not able to achieve appreciable results in terms of classification of terrain data with respect to the sea data. Moreover, the EEF turns out to be the best order selector on this data set, because it is able to show the largest amount of details within the image.

Reflection symmetry (blue pixels) is predominant on the sea area in Fig. 10, whereas the terrain area is classified as $H_{1}$ or $H_{2}$, which means that no symmetry (black pixels) or reflection symmetry is detected; finally, the area separating the sea and terrain areas is mainly classified as azimuthal symmetry (green pixels).

\section{Analysis on Real AIRSAR Data}

To give an additional evidence of the effectiveness of the proposed algorithm, in this section, a second real SAR data set is analyzed, and the results are discussed. In particular, an L-band coherent polarimetric data set ${ }^{5}$ acquired in 1988 from the Jet Propulsion Laboratory (JPL) using an airborne SAR (AIRSAR) on the San Francisco Bay [1], [8] is utilized. The image (900 $\times$ 1024 pixels) contains a mixed urban, vegetation, and sea scene, whose span is reported in Fig. 11(a). To give further information on the considered area, in Fig. 11(b), the corresponding $H-A-\alpha$ decomposition is depicted in RGB colors.

In Fig. 12, the detected symmetries are plotted using the AIC, BIC, GIC (with $\rho=3$ ), and EEF, respectively, with $K=25$,

\footnotetext{
${ }^{3}$ The data can be downloaded at http://uavsar.jpl.nasa.gov.

${ }^{4}$ The specific acquisition is SSurge_15305_14170_007_141120_L090_CX_01.
}

${ }^{5}$ The data can be downloaded at https://earth.esa.int/web/polsarpro/datasources/sample-datasets. 


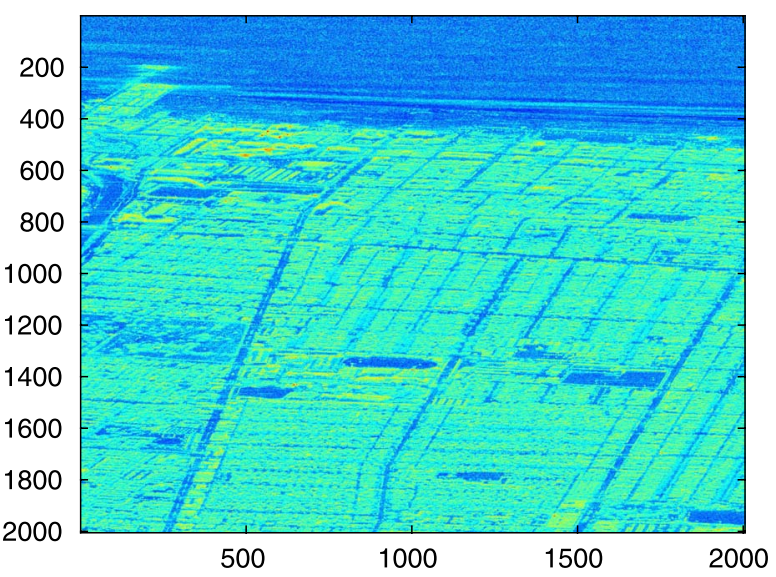

(a) span

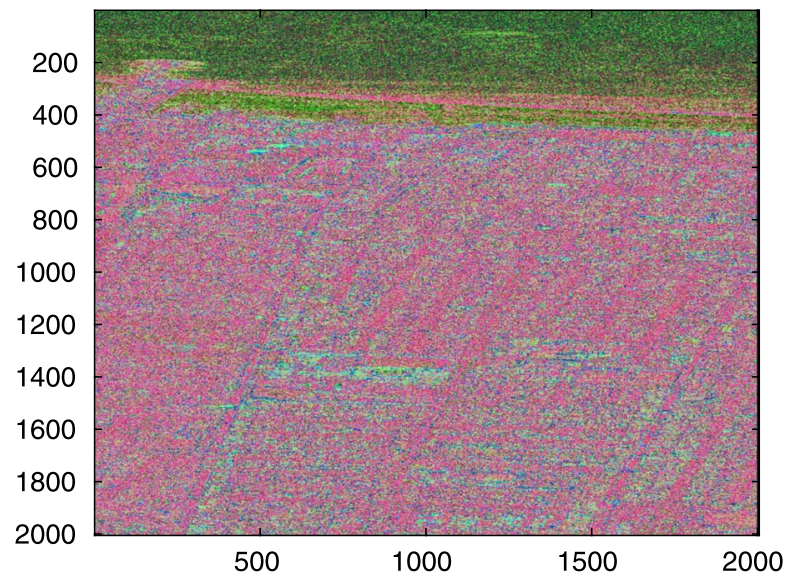

(b) H-A- $\alpha$ decomposition

Fig. 9. Real L-band data UAVSAR pass SSurge_15305_14170_007_141120_L090_CX_01. (a) Span and (b) RGB of the $H$ - $A$ - $\alpha$ decomposition for the reference image of size $2000 \times 2000$ pixels.

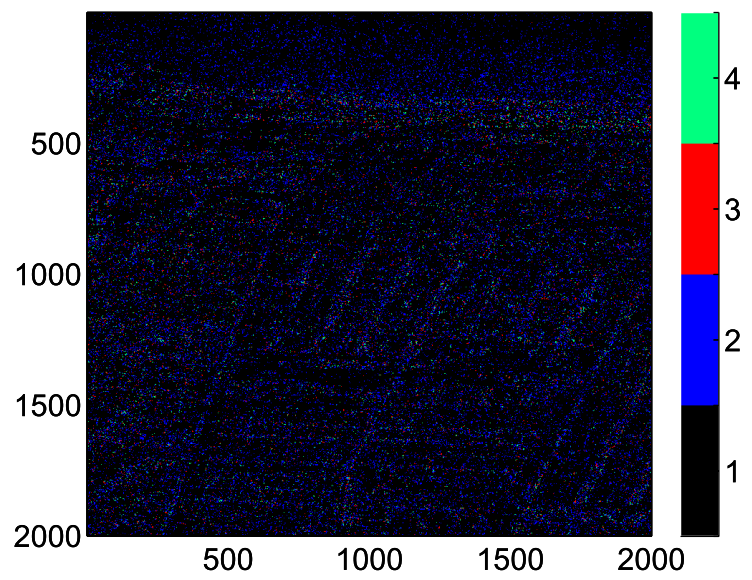

(a)

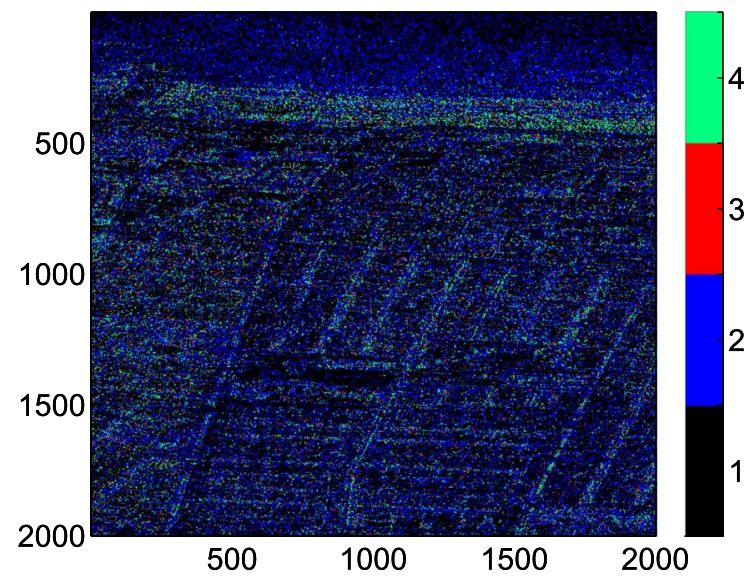

(c)
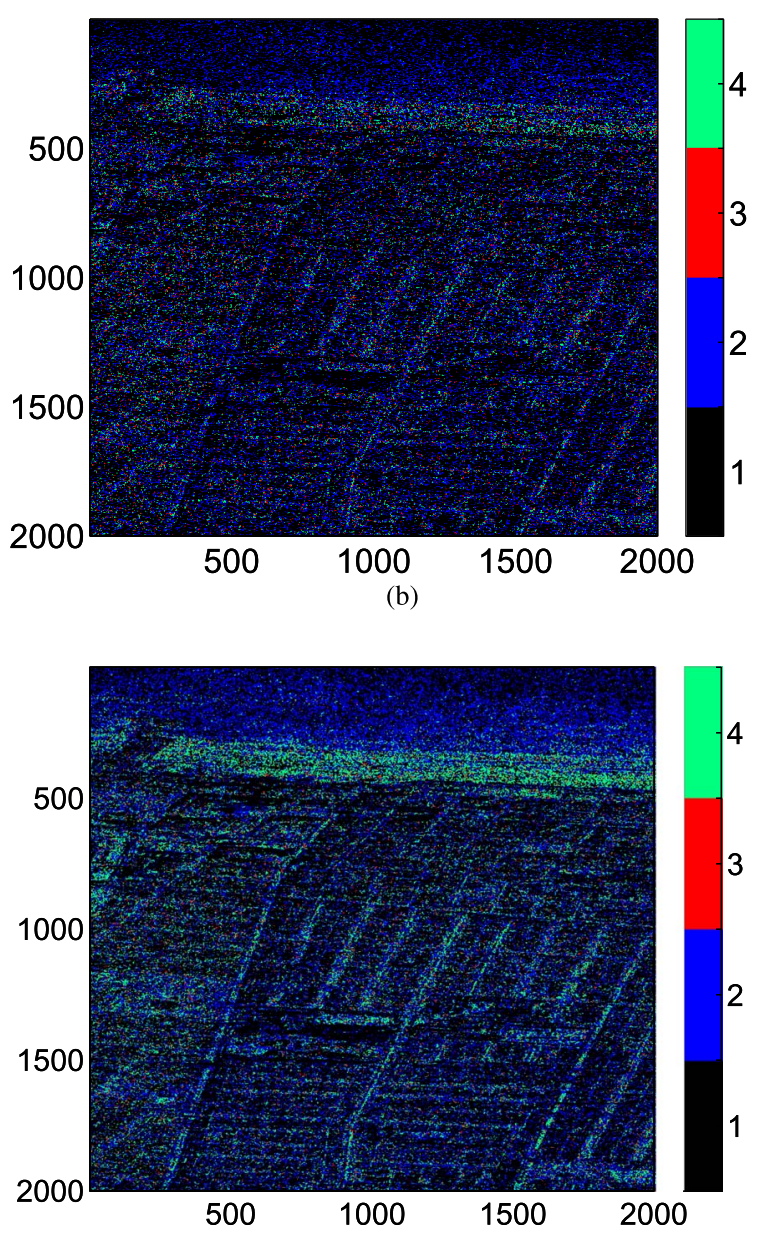

(d)

Fig. 10. Real L-band data UAVSAR pass SSurge_15305_14170_007_141120_L090_CX_01. Detected symmetries within the reference image, $K=25$. (a) AIC. (b) BIC. (c) GIC, $\rho=3$. (d) EEF.

i.e., a $5 \times 5$ sliding window. As before, for each pixel of the considered scene, a specific color corresponds to a specific symmetry class.

The results confirm those obtained with the UAVSAR data: The GIC, BIC, and EEF outperform the AIC. In fact, the different areas are clearly distinguished exploiting the BIC, the GIC, and EEF, whereas for the AIC, some ambiguities arise with reference to the classification of the sea.

However, comparing the BIC, GIC, and EEF assignment results in Fig. 12 with the span image in Fig. 11, it can be 

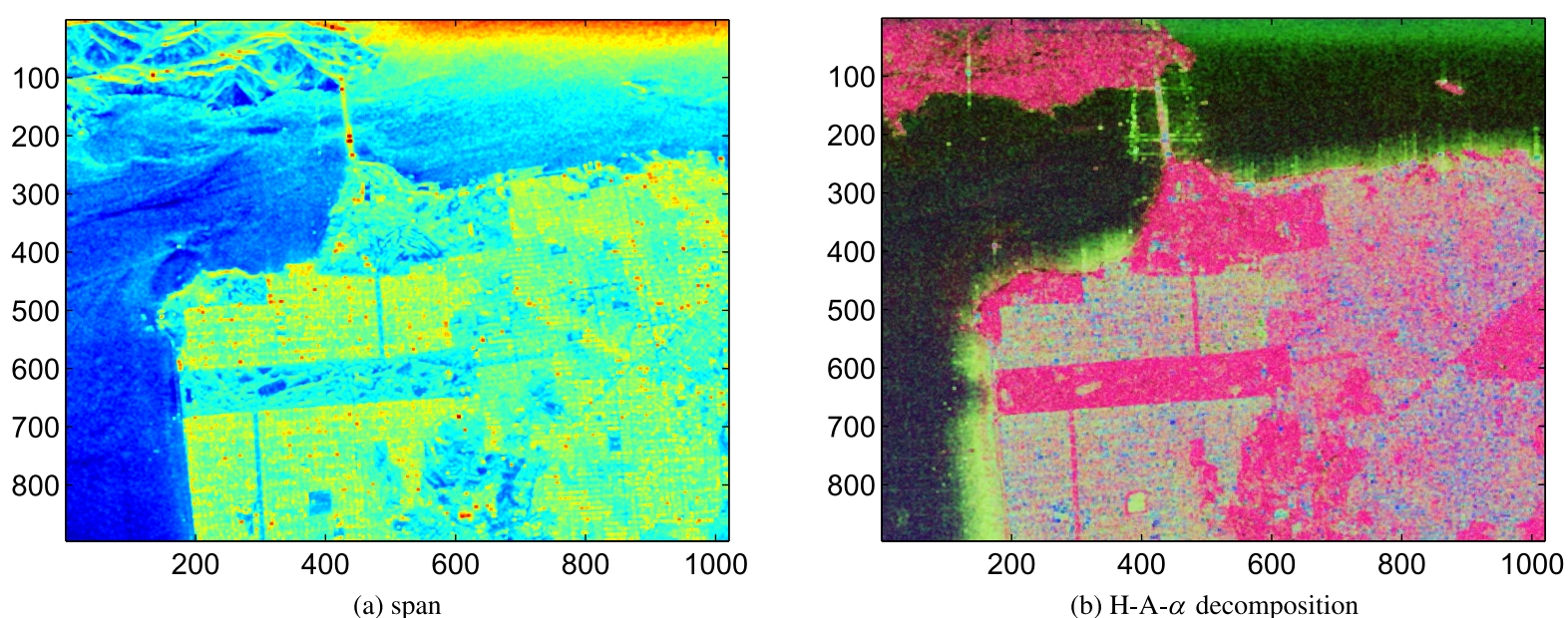

(b) H-A- $\alpha$ decomposition

Fig. 11. San Francisco Bay JPL (AIRSAR L-band 1988) data. (a) Span (in decibels) and (b) RGB of the $H$ - $A$ - $\alpha$ decomposition for the reference image of size $900 \times 1024$ pixels.
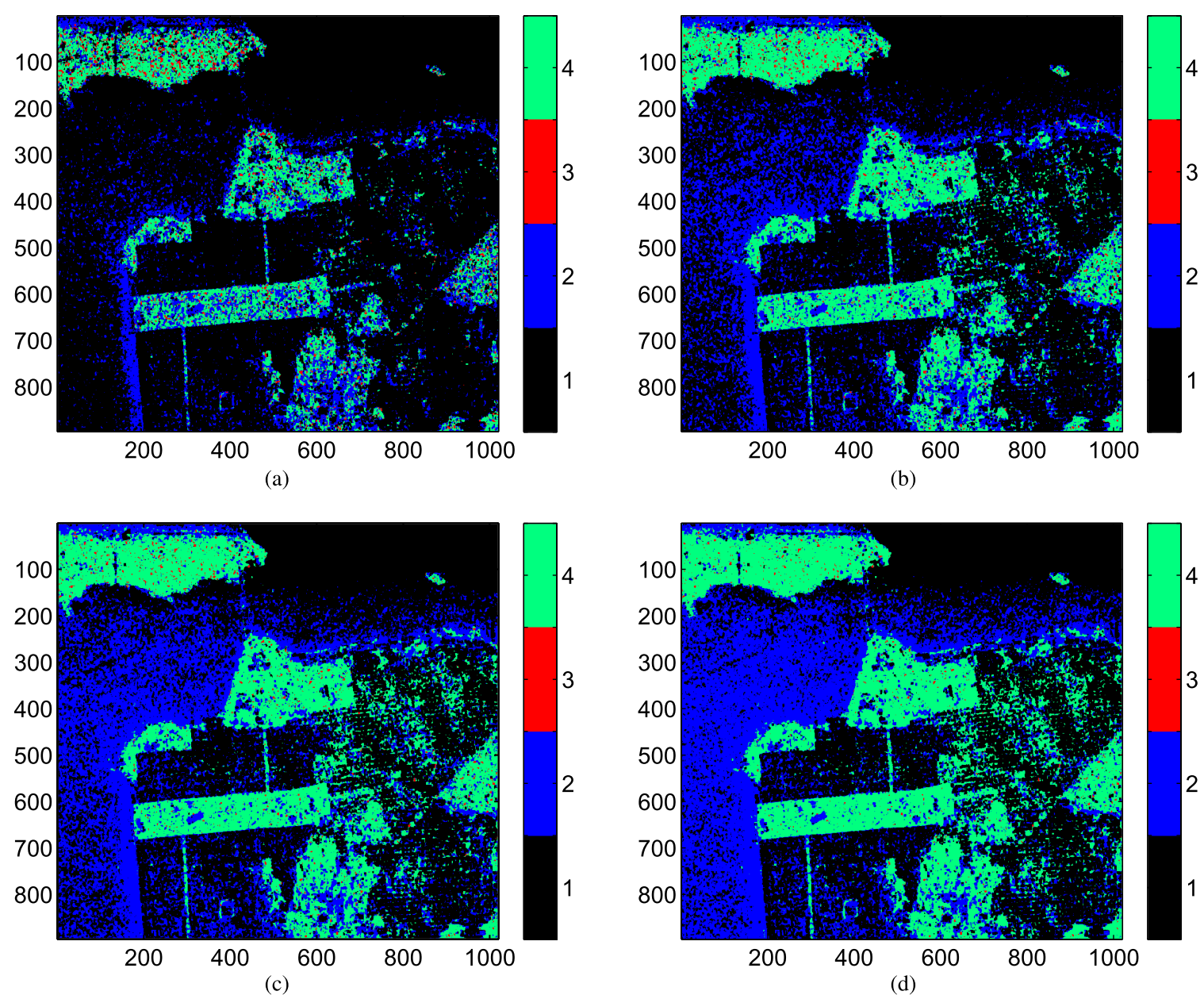

Fig. 12. Real L-band data AIRSAR San Francisco Bay JPL. Detected symmetries within the reference image, $K=25$. (a) AIC. (b) BIC. (c) GIC, $\rho=3$. (d) EEF.

observed that the sea is classified as showing reflection symmetries (blue color). Moreover, the vegetation areas are classified as azimuth symmetric (green color), and the urban scenes are associated to the absence of any symmetry (black pixels). This is probably due to the strong heterogeneity of the environment precluding the rising of a dominant scattering symmetry. As a final remark, the number of pixels classified with rotation symmetry is very small.

To provide a more quantitative analysis, in Table I, the percentage of pixels classified as sharing the same symmetry is given for each order selector. Considering the EEF, the table highlights that the rotation symmetry is almost never 
TABLE I

REAL L-BAND DATA (AIRSAR SAN FRANCISCO BAY JPL). Number of PiXels (IN PERCENTAge OVER the Total) SHARING A SPECIFIC SYMMETRY

\begin{tabular}{ccccc} 
& AIC & BIC & GIC & EEF \\
\hline \hline$H_{1}$ & $74 \%$ & $77 \%$ & $50 \%$ & $46 \%$ \\
\hline$H_{2}$ & $13 \%$ & $12 \%$ & $28 \%$ & $30 \%$ \\
\hline$H_{3}$ & $2 \%$ & $2 \%$ & $1 \%$ & $1 \%$ \\
\hline$H_{4}$ & $11 \%$ & $9 \%$ & $21 \%$ & $23 \%$ \\
\hline
\end{tabular}

observed (only $1 \%$ of the pixels). Otherwise stated, there is a prevalence of the other symmetry classes: The percentage of pixels classified as exhibiting a rotation symmetry is $30 \%$ (the majority of the sea pixels), whereas $23 \%$ are azimuth symmetric pixels mostly belonging to the vegetation areas. The remaining pixels do not exhibit any symmetry and are mainly located in the urban areas and in a particular sea zone where, as illustrated in Fig. 11 (span), there are very strong returns.

\section{Quantitative Comparison Between $H / \alpha$ and Symmetric $H / \alpha$ Classification}

The scope of this section is to provide a quantitative comparison between the classic $H / \alpha$ classification and that exploiting our algorithm as the preprocessing stage (the block diagram illustrating the latter approach is shown in Fig. 13). Specifically, the covariance matrix estimate utilized to perform the $H / \alpha$ decomposition is that provided by the EEF selector in place of the classic sample covariance matrix.

The $H / \alpha$ classification provides as output nine classes that are explicitly defined within [1] and [8]. To have a quantitative comparison, we compute the confusion matrix between the two mentioned classifications assuming as "reference" class that assigned to each pixel by the $H / \alpha$ algorithm, whereas the test outcome is the class assigned by the symmetrical $H / \alpha$ approach.

From the confusion matrix, reported in Table II, it appears that the main difference between the two classifiers is in the selection of class 5 . Specifically, $31.23 \%$ of the pixels that belonged to class 5 according to the $H / \alpha$ classifier are associated to class 2 by the symmetric $H / \alpha$ rule. This is tantamount to classifying pixels as high-entropy vegetation scattering instead of medium-entropy vegetation scattering. From the visual point of view [see Fig. 14(a) and (b)], it appears that the symmetric $H / \alpha$ rule provides more delineated borders of the vegetated region corresponding to the green rectangle located in the middle of the left part of Fig. 14. Moreover, structures corresponding to buildings would result more visible in the image produced by the symmetric $H / \alpha$ classification (i.e., pixels classified as 1 that are representative of double-bounce mechanisms). As an example, the reader could refer to the zones labeled with letters A and B within the images in Fig. 14, where, in the case of the symmetric $H / \alpha$ classifier, the de Young Museum (label A) and the Alhoa Avenue (label B) are more visible with respect to the classic $H / \alpha$ classifier. In fact, with the symmetric algorithm, they are associated to class-1 pixels, which correspond to returns from buildings.

\section{E. Application for Oil Spill Symmetry Characterization}

To further assess the performance of the proposed algorithms and their capabilities to distinguish among different areas within a scene, we apply them to a zone of the GOMoil_07601_10052_101_100622_L090_CX_02 SAR image that is composed of sea data containing also an oil spill. The image has been acquired on June 22, 2010, during the British Petroleum oil spill incident in the Gulf of Mexico (known also as the Deepwater Horizon Oil Spill). As in the previous analysis, this second image contains a scene acquired with all the polarizations, and the corresponding polarimetric overlay is reported in Fig. 15. Again, the selected area of interest is a subimage of $2000 \times 2000$ pixels, whose span is displayed in Fig. 16(a) and its $H-A$ - $\alpha$ decomposition in RGB colors in Fig. 16(b). This choice is, of course, for testing our techniques as well as to provide some suggestions on possible applications of the algorithm in real operative contexts.

We represent in Fig. 17 the detected symmetries for the reference image, computed using, respectively, the AIC, BIC, GIC (with $\rho=3$ ), and EEF, with $K=25$.

The results show that both the AIC and the BIC achieve quite good results in terms of classification of sea data with respect to oil spills, since the sea pixels are classified as reflection symmetry (blue pixels), whereas the oil spill shows some azimuthal symmetry (green pixels) in the radar returns. However, both the GIC and EEF outperform the AIC and BIC, with a clear separation of the different regions within the area under test. It is also interesting to observe a small strip characterized by an azimuthal symmetry (green pixels) in correspondence of a small ship transition over the sea (see the ellipse in Fig. 17).

\section{CONCLusion}

We have introduced and analyzed a new framework for detecting covariance symmetries within polarimetric SAR images. The proposed algorithm is based on the exploitation of the special structures assumed by the polarimetric coherence matrix whenever symmetrical properties of the returns associated to the pixels under analysis occur. Specifically, the core of the technique is the utilization of model order selectors, capitalizing on the coherence matrix structures, to classify each pixel of the considered scene.

The analysis of the new technique has been conducted on both simulated and real SAR data. In particular, the former has shown good capabilities in correctly classifying the data in a controlled environment. Moreover, the latter has demonstrated the effectiveness of the approach and its flexibility to be integrated in operative context and algorithms, such as oil spill detections.

Future research work might concern the analysis of the new technique on other available real radar data sets such as AgriSar data [30], as well as its integration in more complex algorithms to produce land cover classifications. Moreover, another possible future work, very suitable for spaceborne applications, might concern the introduction of a fifth hypothesis $\left(H_{5}\right)$, accounting for the presence of acquisition noise only. In this case, if the algorithm decides for $H_{5}$, no further processing is possible since no real information on the scene is available. 


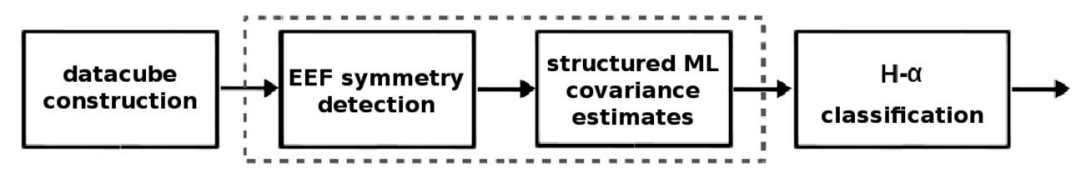

Fig. 13. Block scheme of the symmetric $H$ - $\alpha$ classification.

TABLE II

REAL L-BAND DATA (AIRSAR SAN FRANCISCO BAY JPL). CONFUSION Matrix (EXPRessed in PerCentage) Between the Classic $H / \alpha$ ClasSification AND THAT EXPLOITING OUR ALGORITHM AS Preprocessing STAGE. THE VALUES IN THE TABle are EXPREsSed in Percentage

\begin{tabular}{|c|c|c|c|c|c|c|c|c|c|}
\hline \multicolumn{10}{|c|}{ Symmetric $\mathbf{H} / \alpha$} \\
\hline & 1 & 2 & \begin{tabular}{|l|}
3 \\
\end{tabular} & 4 & 5 & 6 & 7 & 8 & 9 \\
\hline 1 & 99.49 & 0.51 & 0 & 0 & 0 & 0 & 0 & 0 & 0 \\
\hline 2 & 10.03 & 89.97 & 0 & 0 & 0 & 0 & 0 & 0 & 0 \\
\hline 3 & 0 & 0 & \begin{tabular}{|l|l|}
0 \\
\end{tabular} & 0 & 0 & 0 & 0 & 0 & 0 \\
\hline $\bar{\Omega}$ & 4.10 & 8.41 & 0 & 87.48 & 0.01 & 0 & 0 & 0 & 0 \\
\hline 5 & 0 & 31.23 & 0 & 3.76 & 58.10 & 6.91 & 0 & 0 & 0 \\
\hline 6 & 0 & 0 & 0 & 0 & 1.78 & 98.22 & 0 & 0 & 0 \\
\hline 7 & 0 & 0 & 0 & 0.43 & 0.01 & 0 & 99.56 & 0 & 0 \\
\hline 8 & 0 & 0 & 0 & 0 & 5.74 & 0 & 0 & 94.26 & 0 \\
\hline 9 & 0 & 0 & 0 & 0 & 0.01 & 0.85 & 0 & 0 & 99.14 \\
\hline
\end{tabular}

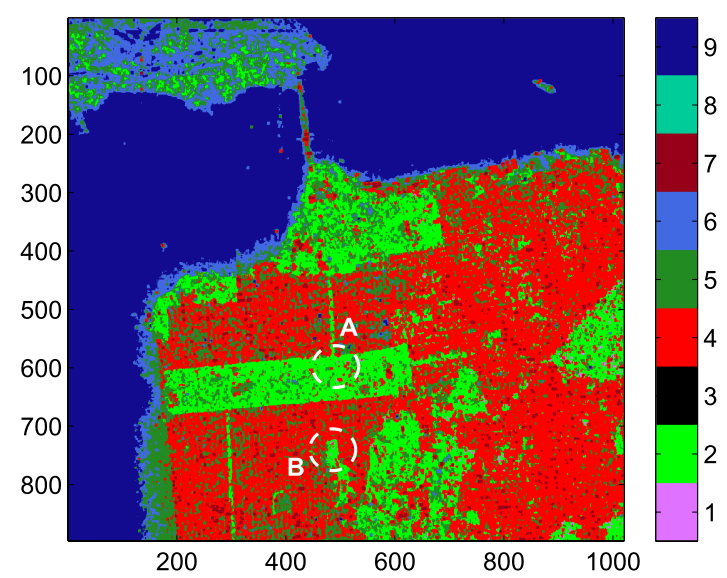

(a)

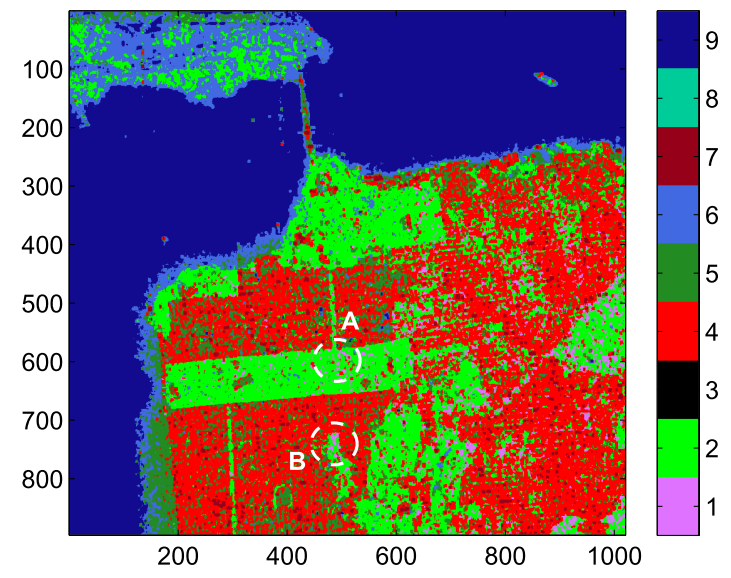

(b)

Fig. 14. Real L-band data (AIRSAR San Francisco Bay JPL). $H$ - $\alpha$ classification. (a) $H-\alpha$ classifier. (b) Symmetric $H$ - $\alpha$ classifier.

\section{APPENDIX}

\section{A. Proof of Proposition 3.2}

We show how the minimization problem (11) can be recast in the presence of the considered symmetry properties, exploiting

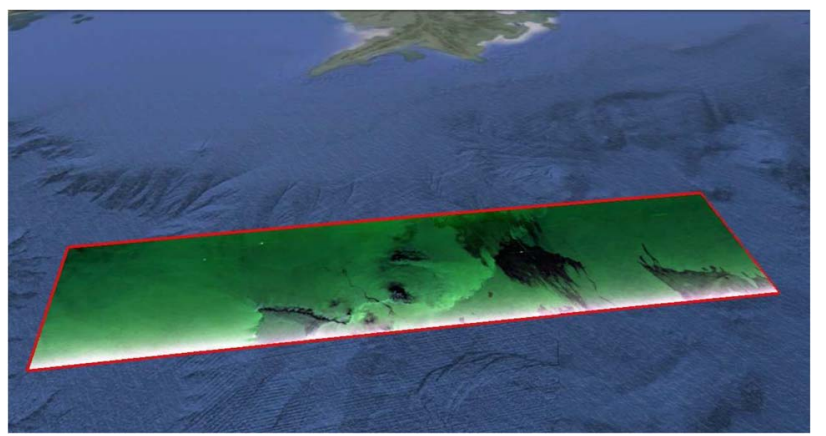

Fig. 15. Three-polarization color overlay of the SAR image GOMoil_07601_10052_101_100622_L090_CX_02.

the results given by Lemma 3.1. Specifically, in the case of reflection symmetry, the minimization problem (11) can be simplified as follows:

$$
\begin{aligned}
& \min _{\boldsymbol{C} \in \mathcal{H}_{3}^{++}}\left[\log \operatorname{det}(\boldsymbol{C})+\operatorname{tr}\left(\boldsymbol{C}^{-1} \boldsymbol{S}\right)\right] \\
& =\min _{\boldsymbol{C}_{1} \in \mathcal{H}_{2}^{++}}\left[\log \operatorname{det}\left(\boldsymbol{C}_{1}\right)+\operatorname{tr}\left(\boldsymbol{C}_{1}^{-1} \overline{\boldsymbol{S}}_{1,1}\right)\right] \\
& \quad+\min _{c>0}\left[\log (c)+\frac{\bar{S}_{3,3}}{c}\right] \\
& =\log \operatorname{det}\left(\overline{\boldsymbol{S}}_{1,1}\right)+\log \left(\bar{S}_{3,3}\right)+3 .
\end{aligned}
$$

Moreover, for the case of rotation symmetry, the objective function reduces to

$$
\begin{aligned}
\log \operatorname{det}(\boldsymbol{C})+\operatorname{tr}\left(\boldsymbol{C}^{-1} \boldsymbol{S}\right) \\
=\log \operatorname{det}\left(\boldsymbol{V} \boldsymbol{E} \boldsymbol{T} \boldsymbol{C} \boldsymbol{T}^{\dagger} \boldsymbol{E} \boldsymbol{V}^{\dagger}\right)-2 \log \operatorname{det}(\boldsymbol{E}) \\
\quad+\operatorname{tr}\left\{\left(\boldsymbol{V} \boldsymbol{E} \boldsymbol{T} \boldsymbol{C} \boldsymbol{T}^{\dagger} \boldsymbol{E} \boldsymbol{V}^{\dagger}\right)^{-1}\left(\boldsymbol{V} \boldsymbol{E} \boldsymbol{T} \boldsymbol{S} \boldsymbol{T}^{\dagger} \boldsymbol{E} \boldsymbol{V}^{\dagger}\right)\right\} \\
=\log \operatorname{det}\left(\boldsymbol{C}_{2}\right)+\log (a)-2 \log \operatorname{det}(\boldsymbol{E})+\operatorname{tr}\left(\boldsymbol{C}_{2}^{-1} \tilde{\boldsymbol{S}}_{2}\right)+\frac{\tilde{S}_{1,1}}{a} \\
=\log \operatorname{det}\left(\boldsymbol{C}_{2}\right)+\log (a)+\log (2)+\operatorname{tr}\left(\boldsymbol{C}_{2}^{-1} \tilde{\boldsymbol{S}}_{2}\right)+\frac{\tilde{S}_{1,1}}{a} .
\end{aligned}
$$

Consequently, the minimization problem (11) can be expressed as follows:

$$
\begin{aligned}
& \min _{\boldsymbol{C} \in \mathcal{H}_{3}^{++}}\left[\log \operatorname{det}(\boldsymbol{C})+\operatorname{tr}\left(\boldsymbol{C}^{-1} \boldsymbol{S}\right)\right] \\
& =\min _{\boldsymbol{C}_{2} \in \mathcal{S}_{2}^{++} \cap \mathcal{P}_{2}^{++}}\left[\log \operatorname{det}\left(\boldsymbol{C}_{2}\right)+\operatorname{tr}\left(\boldsymbol{C}_{2}^{-1} \tilde{\boldsymbol{S}}_{2,2}\right)\right] \\
& \quad+\log \left(\tilde{S}_{1,1}\right)+1+\log (2) \\
& =\min _{\boldsymbol{C}_{2} \in \mathcal{S}_{2}^{++} \cap \mathcal{P}_{2}^{++}}\left[\log \operatorname{det}\left(\boldsymbol{C}_{2}\right)\right. \\
& \left.\quad+\operatorname{tr}\left(\frac{\boldsymbol{C}_{2}^{-1}}{2}\left(\tilde{\boldsymbol{S}}_{2,2}+\boldsymbol{J} \tilde{\boldsymbol{S}}_{2,2} \boldsymbol{J}\right)\right)\right] \\
& \quad+\log \left(\tilde{S}_{1,1}\right)+1+\log (2) \\
& =\log \operatorname{det}\left[\frac{1}{2}\left(\tilde{\boldsymbol{S}}_{2,2}+\boldsymbol{J} \tilde{\boldsymbol{S}}_{2,2} \boldsymbol{J}\right)\right]+\log \left(\tilde{S}_{1,1}\right)+3+\log (2)
\end{aligned}
$$




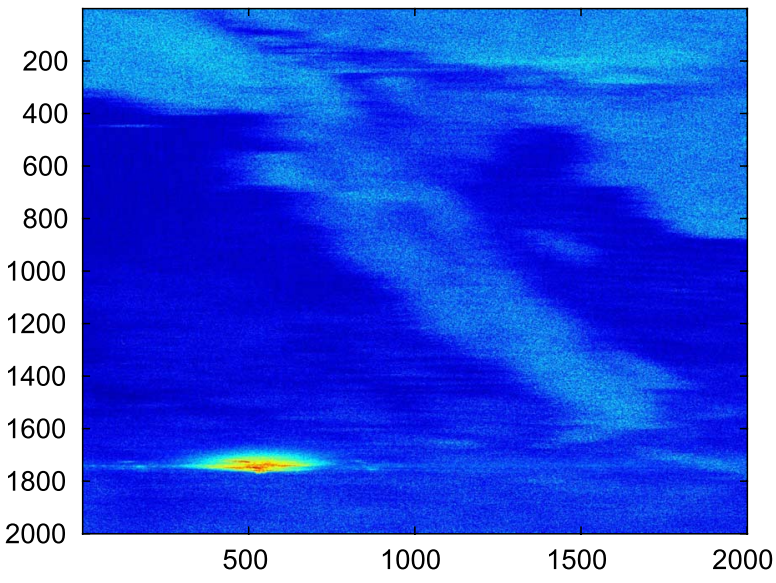

(a) span

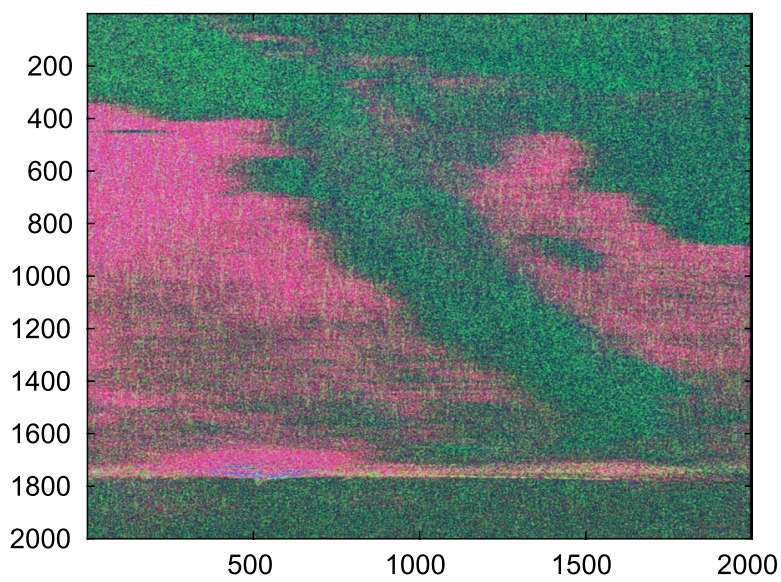

(b) H-A- $\alpha$ decomposition

Fig. 16. Real L-band data SAR image GOMoil_07601_10052_101_100622_L090_CX_02. (a) Span (in decibels) and (b) RGB of the $H$ - $A$ - $\alpha$ decomposition for the reference image of size $2000 \times 2000$ pixels.

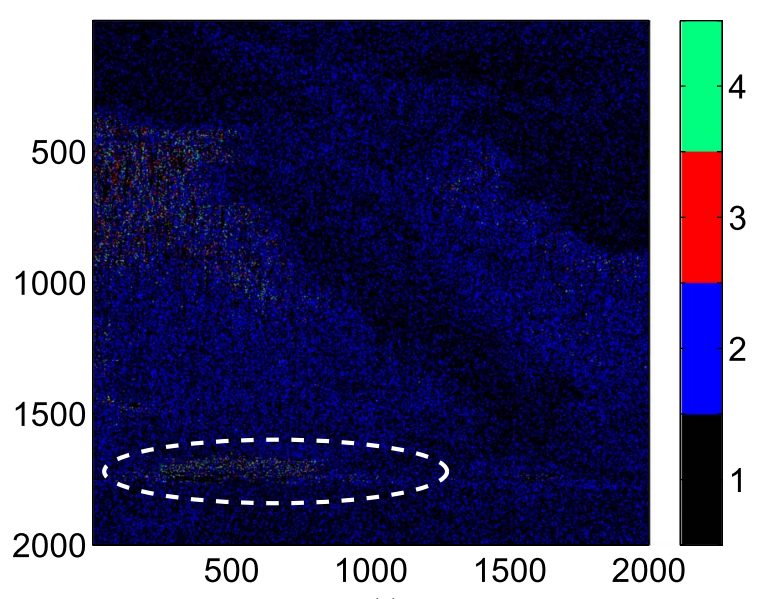

(a)

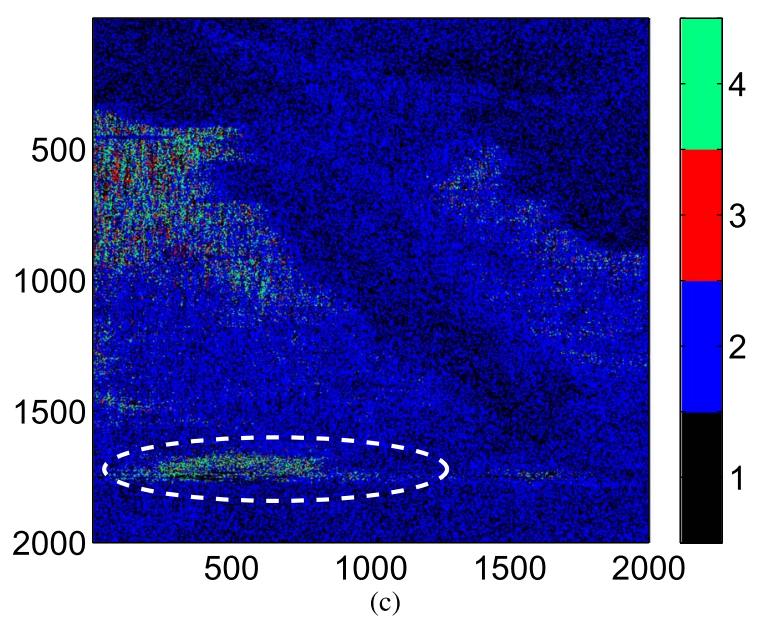

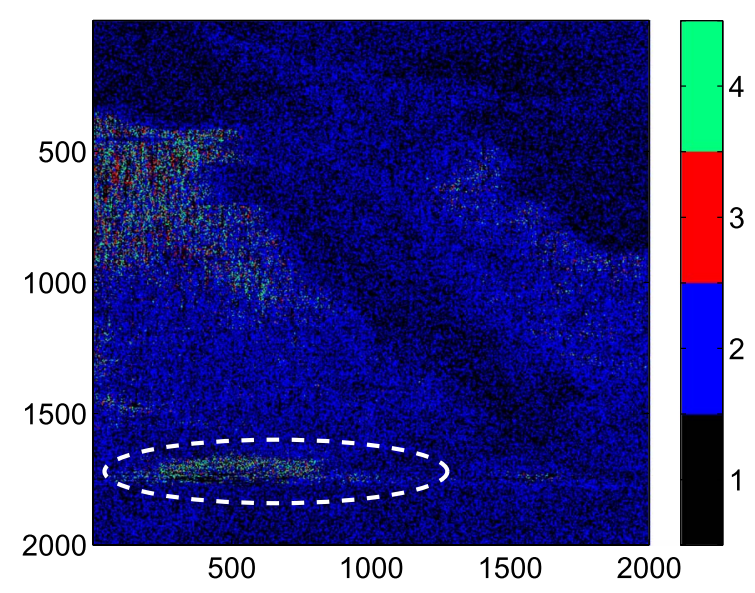

(b)

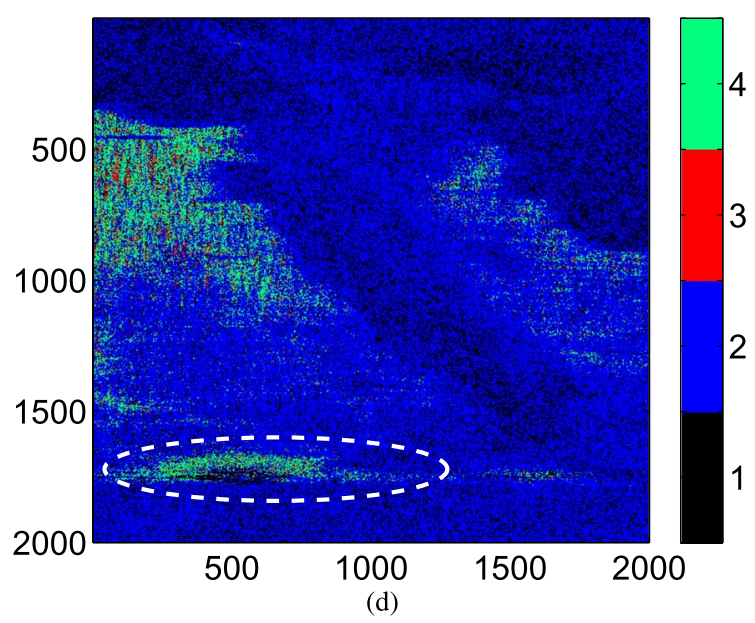

Fig. 17. Real L-band data SAR image GOMoil_07601_10052_101_100622_L090_CX_02. Detected symmetries within the reference image, $K=25$. (a) AIC. (b) BIC. (c) GIC, $\rho=3$. (d) EEF.

where in the second equality, the persymmetric and real sym- and hence metric structure of $\boldsymbol{C}_{2}$ is used to claim

$$
\operatorname{tr}\left(\boldsymbol{C}_{2}^{-1} \tilde{\boldsymbol{S}}_{2,2}\right)=\operatorname{tr}\left(\boldsymbol{C}_{2}^{-1} \boldsymbol{J} \tilde{\boldsymbol{S}}_{2,2} \boldsymbol{J}\right) \quad \operatorname{tr}\left(\boldsymbol{C}_{2}^{-1} \tilde{\boldsymbol{S}}_{2,2}\right)=\operatorname{tr}\left(\boldsymbol{C}_{2}^{-1} \frac{1}{2}\left(\tilde{\boldsymbol{S}}_{2,2}+\boldsymbol{J} \tilde{\boldsymbol{S}}_{2,2} \boldsymbol{J}\right)\right)
$$


Finally, for the case of azimuth symmetry, the following equalities hold:

$$
\begin{aligned}
\log \operatorname{det}(\boldsymbol{C})+\operatorname{tr}\left(\boldsymbol{C}^{-1} \boldsymbol{S}\right) \\
=\log \operatorname{det}\left(\boldsymbol{E} \boldsymbol{T C} \boldsymbol{T}^{\dagger} \boldsymbol{E}\right)-2 \log \operatorname{det}(\boldsymbol{E}) \\
\quad+\operatorname{tr}\left\{\left(\boldsymbol{E} \boldsymbol{T C} \boldsymbol{T}^{\dagger} \boldsymbol{E}\right)^{-1}\left(\boldsymbol{E} \boldsymbol{T} \boldsymbol{S} \boldsymbol{T}^{\dagger} \boldsymbol{E}\right)\right\} \\
=\log (a)+2 \log (b)+\log (2)+\frac{\hat{S}_{1,1}}{a}+\frac{\hat{S}_{2,2}+\hat{S}_{3,3}}{b}
\end{aligned}
$$

and the minimization problem (11) can be rewritten as

$$
\begin{aligned}
\min _{\boldsymbol{C} \in \mathcal{H}_{3}^{++}} & {\left[\log \operatorname{det}(\boldsymbol{C})+\operatorname{tr}\left(\boldsymbol{C}^{-1} \boldsymbol{S}\right)\right] } \\
& =\log \left(\hat{S}_{1,1}\right)+2 \log \left(\frac{\hat{S}_{2,2}+\hat{S}_{3,3}}{2}\right)+3+\log (2) .
\end{aligned}
$$

\section{B. Computation of $\boldsymbol{F}$ and Regularity Conditions}

We verify, for the BIC rule, the regularity conditions that must hold on the Fisher information matrix $\boldsymbol{F}$ :

1) Invertibility of the Fisher Information Matrix: The ML estimator of the parameter vector (synthetically denoted by $\hat{\boldsymbol{\theta}}$ ) is unbiased and with finite variance. Hence, by [31, Eq. (18)], applied with $\boldsymbol{H}=\boldsymbol{I}$

$$
\boldsymbol{I}=\boldsymbol{F} \boldsymbol{F}^{+}
$$

must hold. This is true if and only if $\boldsymbol{F}$ is invertible.

2) Regularity Condition: $\boldsymbol{F} / K=O(1)$ : By Slepian-Bangs formula of [32, p. 927]

$$
F_{i, j}=K \operatorname{tr}\left[\boldsymbol{C}^{-1}(\hat{\boldsymbol{\theta}}) \frac{\partial \boldsymbol{C}(\boldsymbol{\theta})}{\partial \theta_{i}} \boldsymbol{C}^{-1}(\hat{\boldsymbol{\theta}}) \frac{\partial \boldsymbol{C}(\boldsymbol{\theta})}{\partial \theta_{j}}\right] .
$$

Since $\boldsymbol{C}(\hat{\theta})$ tends to the true covariance as $K \rightarrow \infty$, whereas the terms $A_{i}=\partial \boldsymbol{C}(\boldsymbol{\theta}) / \partial \theta_{i}$ and $A_{j}=\partial \boldsymbol{C}(\boldsymbol{\theta}) / \partial \theta_{j}$ do not depend on $K$, we have

$$
\frac{F_{i, j}}{K} \rightarrow \operatorname{tr}\left[\boldsymbol{C}(\boldsymbol{\theta})^{-1} A_{i} \boldsymbol{C}(\boldsymbol{\theta})^{-1} A_{j}\right]=O(1) .
$$

\section{ACKNOWLEDGMENT}

The authors would like to thank the Editor and the Referees for the interesting questions and comments that have helped improve this paper. They would also like to thank NASA/JPL for providing the UAVSAR data and ESA for the AIRSAR data. All data supporting this paper are available from http://uavsar.jpl. nasa.gov and https://earth.esa.int/web/polsarpro/data-sources/ sample-datasets.

\section{REFERENCES}

[1] J. S. Lee and E. Pottier, Polarimetric Radar Imaging: From Basics to Applications. Boca Raton, FL, USA: CRC Press, 2009.

[2] S. V. Nghiem, S. H. Yueh, R. Kwok, and F. K. Li, "Symmetry properties in polarimetric remote sensing," Radio Sci., vol. 27, no. 5, pp. 693-711, Sep./Oct. 1992.
[3] L. Ferro-Famil and E. Pottier, "Dual frequency polarimetric SAR data classification and analysis," in Progress In Electromagnetics Research, vol. 24, J. A. Kong, Ed. New York, NY, USA: Elsevier, 2001, pp. 251-276.

[4] J. C. Curlander and R. N. McDonough, Synthetic Aperture Radar. New York, NY, USA: Wiley, 1991.

[5] S. R. Cloude and E. Pottier, "A review of target decomposition theorems in radar polarimetry," IEEE Trans. Geosci. Remote Sens., vol. 34, no. 2, pp. 498-518, Mar. 1996.

[6] E. Krogager, "New decomposition of the radar target scattering matrix," Electron. Lett., vol. 26, no. 18, pp. 1525-1527, 1990.

[7] W. L. Cameron and L. K. Leung, "Feature motivated polarization scattering matrix decomposition," in Proc. IEEE Int. Radar Conf., Arlington, VA, USA, May 7-10, 1990, pp. 549-557.

[8] S. R. Cloude and E. Pottier, "An entropy based classification scheme for land applications of polarimetric SAR," IEEE Trans. Geosci. Remote Sens., vol. 35, no. 1, pp. 68-78, Jan. 1997.

[9] J. J. V. Zyl, "Unsupervised classification of scattering behavior using radar polarimetry data," IEEE Trans. Geosci. Remote Sens., vol. 27, no. 1, pp. 36-45, Jan. 1989.

[10] S. E. Park, W. M. Moon, and E. Pottier, "Assessment of scattering mechanism of polarimetric SAR signal from mountainous forest areas," IEEE Trans. Geosci. Remote Sens., vol. 50, no. 11, pp. 4711-4719, Nov. 2012.

[11] M. Migliaccio, F. Nunziata, A. Montuori, L. Xiaofeng, and W. G. Pichel, "A multifrequency polarimetric SAR processing chain to observe oil fields in the Gulf of Mexico," IEEE Trans. Geosci. Remote Sens., vol. 49, no. 12, pp. 4729-4737, Dec. 2011.

[12] A. B. Salberg, O. Rudjord, and A. H. S. Solberg, "Oil spill detection in hybrid-polarimetric SAR images," IEEE Trans. Geosci. Remote Sens., vol. 52, no. 10, pp. 6521-6533, Oct. 2014.

[13] S. Mermoz, S. Allain-Bailhache, M. Bernier, E. Pottier, J. J. van der Sanden, and K. Chokmani, "Retrieval of river ice thickness from C-band PolSAR data," IEEE Trans. Geosci. Remote Sens., vol. 52, no. 6, pp. 3052-3062, Jun. 2014.

[14] G. Zhou, Y. Cui, Y. Chen, J. Yang, H. Rashvand, and Y. Yamaguchi, "Linear feature detection in polarimetric SAR images," IEEE Trans. Geosci. Remote Sens., vol. 49, no. 4, pp. 1453-1463, Apr. 2011.

[15] S. M. Kay, Fundamentals of Statistical Signal Processing: Estimation Theory, vol. 1. Englewood Cliffs, NJ, USA: Prentice-Hall, 1993.

[16] S. M. Kay, "The multifamily likelihood ratio test for multiple signal model detection," IEEE Signal Process. Lett., vol. 12, no. 5, pp. 369-371, May 2005.

[17] Y. Selén, "Model selection," Ph.D. dissertation, Electr. Eng., Uppsala Univ., Uppsala, Sweden, Oct. 2004.

[18] P. Stoica and Y. Selén, "Model-order selection: A review of information criterion rules," IEEE Signal Process. Mag., vol. 21, no. 4, pp. 36-47, Apr. 2004.

[19] P. Stoica, Y. Selén, and J. Li, "On information criteria and the generalized likelihood ratio test of model order selection," IEEE Signal Process. Lett., vol. 11, no. 10, pp. 794-797, Oct. 2004.

[20] S. M. Kay, "Exponentially embedded families-New approaches to model order estimation," IEEE Trans. Aerosp. Electron. Syst., vol. 41, no. 1, pp. 333-345, Jan. 2005.

[21] F. Bordoni, F. Gini, and L. Verrazzani, "Capon-LS for model order selection of multicomponent interferometric SAR signals," Proc. Inst. Elect. Eng.—Radar, Sonar Navigat., vol. 151, no. 5, pp. 299-305, Oct. 2004.

[22] F. Lombardini, L. Rößing, J. Ender, and F. Cai, "Interferometric model order selection: Validation of ITC methods with airborne three-antenna SAR data," in Proc. IEEE IGARSS, 2006, pp. 2565-2568.

[23] S. Sauer, L. Ferro-Famil, A. Reigber, and E. Pottier, "Physical Parameter Extraction Over Urban Areas Using L-band POLSAR Data and Interferometric Baseline Diversity," in Proc. IEEE IGARSS, 2007, pp. 5045-5048.

[24] F. Lombardini and F. Gini, "Model order selection in multi-baseline interferometric radar systems," EURASIP J. Appl. Signal Process., vol. 20, no. 20 , pp. 3206-3219, 2005.

[25] A. Pauciullo, D. Reale, A. D. Maio, and G. Fornaro, "Detection of double scatterers in SAR tomography," IEEE Trans. Geosci. Remote Sens., vol. 50, no. 9, pp. 3567-3586, Sep. 2012.

[26] E. Conte and M. Longo, "Characterisation of radar clutter as a spherically invariant random process," Proc. Inst. Elect. Eng.-Commun., Radar Signal Process., vol. 134, no. 2, pp. 191-197, Apr. 1987.

[27] M. Greco and F. Gini, "Statistical analysis of high-resolution SAR ground clutter data," IEEE Trans. Geosci. Remote Sens., vol. 45, no. 3, pp. 566-575, Mar. 2007.

[28] C. Oliver and S. Quegan, Understanding Synthetic Aperture Radar Images. Raleigh, NC, USA: SciTech, 2004. 
[29] S. Hensley et al., "The UAVSAR instrument: Description and first results," in Proc. IEEE Int. Radar Conf., May 2008, pp. 1-6.

[30] G. D. Martino, A. Iodice, A. Natale, and D. Riccio, "Polarimetric two-scale two-component model for the retrieval of soil moisture under moderate vegetation via L-band SAR data," IEEE Trans. Geosci. Remote Sens., vol. 54, no. 4, pp. 2470-2491, Apr. 2016.

[31] P. Stoica and T. L. Marzetta, "Parameter estimation problems with singular information matrices," IEEE Trans. Signal Process., vol. 49, no. 1, pp. 87-90, Jan. 2001.

[32] H. L. V. Trees, Detection, Estimation and Modulation Theory: Optimum Array Processing. $\quad$ New York, NY, USA: Wiley, 2002.

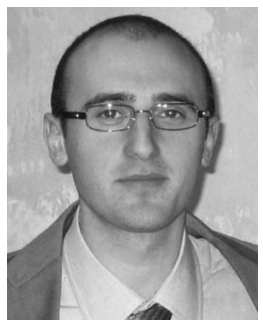

Luca Pallotta (S'12-M'15) received the M.Sc. degree (cum laude) in telecommunication engineering from the University of Sannio, Benevento, Italy, in 2009 and the Ph.D. degree in electronic and telecommunication engineering from the University of Naples Federico II, Naples, Italy, in 2014.

His research interest lies in the field of statistical signal processing, with emphasis on radar signal processing, radar target classification, and SAR image classification.

Dr. Pallotta won the Student Paper Competition at the 2013 IEEE Radar Conference.

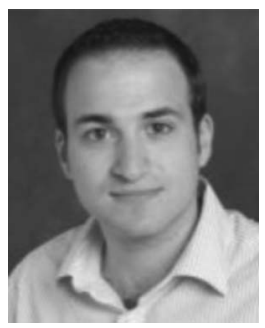

Carmine Clemente (S'09-M'13) received the B.Sc. and M.Sc. degrees (cum laude) in telecommunications engineering from Università degli Studi del Sannio, Benevento, Italy, in 2006 and 2009, respectively, and the Ph.D. degree from the University of Strathclyde, Glasgow, U.K., in 2012.

$\mathrm{He}$ is currently a Lecturer with the Department of Electronic and Electrical Engineering, University of Strathclyde, working on advanced radar signal processing algorithms, MIMO radar systems, and micro-Doppler analysis. His research interests include SAR focusing and bistatic SAR focusing algorithm development, microDoppler signature analysis and extraction from multistatic radar platforms, micro-Doppler classification, and statistical signal processing.

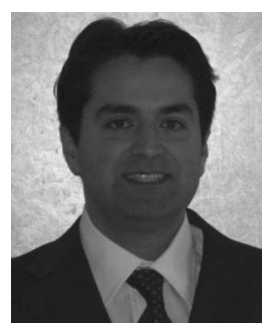

Antonio De Maio (S'01-A'02-M'03-SM'07-F'13) was born in Sorrento, Italy, on June 20, 1974 He received the Dr.Eng. degree (with honors) and the Ph.D. degree in information engineering from the University of Naples Federico II, Naples, Italy, in 1998 and 2002, respectively.

From October to December 2004, he was a Visiting Researcher with the U.S. Air Force Research Laboratory, Rome, NY, USA. From November to December 2007, he was a Visiting Researcher with the Chinese University of Hong Kong, Hong Kong. He is currently a Professor with the University of Naples Federico II. His research interest lies in the field of statistical signal processing, with emphasis on radar detection, optimization theory applied to radar signal processing, and multiple-access communications.

Dr. De Maio was a recipient of the 2010 IEEE Fred Nathanson Memorial Award as the young (less than 40 years of age) Aerospace and Electronic Systems Society Radar Engineer for 2010, whose performance is particularly noteworthy as evidenced by contributions to the radar art over a period of several years, with the following citations for "robust constant false alarm rate detection, knowledge-based radar signal processing, and waveform design and diversity."

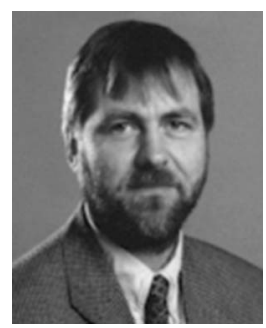

John J. Soraghan (S'83-M'84-SM'96) received the B.Eng. (Hons.) and M.Eng.Sc. degrees from University College Dublin, Dublin, Ireland, in 1978 and 1983, respectively, and the Ph.D. degree from the University of Southampton, Southampton, U.K. in 1989, all in electronic engineering. His doctoral research focused on synthetic aperture radar processing on the distributed array processor.

After graduating, he worked with the Electricity Supply Board in Ireland and with Westinghouse Electric Corporation in the United States. In 1986, he joined the Department of Electronic and Electrical Engineering, University of Strathclyde, Glasgow, U.K., as a Lecturer and became a Senior Lecturer in 1990, a Reader in 2000, and a Professor in signal processing in September 2003, within the Institute for Communications and Signal Processing (ICSP). In December 2005, he became the Head of the ICSP. He currently holds the Texas Instruments Chair in Signal Processing with the University of Strathclyde. He was a Manager of the Scottish Transputer Centre from 1988 to 1991 and a Manager of the DTI Parallel Signal Processing Centre from 1991 to 1995. His main research interests include signal processing theories, algorithms, and architectures with applications to remote sensing, telecommunications, biomedicine, and condition monitoring.

Dr. Soraghan is a member of the Institution of Engineering and Technology. 